\title{
Approximate Solutions by Truncated Taylor Series Expansions of Nonlinear Differential Equations and Related Shadowing Property with Applications
}

\author{
M. De la Sen, ${ }^{1}$ A. Ibeas, ${ }^{2}$ and R. Nistal ${ }^{1}$ \\ ${ }^{1}$ Institute of Research and Development of Processes, University of the Basque Country, Campus of Leioa (Bizkaia), \\ P.O. Box 644, Bilbao, Barrio Sarriena, 48940 Leioa, Spain \\ ${ }^{2}$ Department of Telecommunications and Systems Engineering, Universitat Autònoma de Barcelona, UAB, 08193 Barcelona, Spain
}

Correspondence should be addressed to M. De la Sen; manuel.delasen@ehu.es

Received 5 December 2013; Accepted 11 June 2014; Published 7 July 2014

Academic Editor: Samir Saker

Copyright (c) 2014 M. De la Sen et al. This is an open access article distributed under the Creative Commons Attribution License, which permits unrestricted use, distribution, and reproduction in any medium, provided the original work is properly cited.

\begin{abstract}
This paper investigates the errors of the solutions as well as the shadowing property of a class of nonlinear differential equations which possess unique solutions on a certain interval for any admissible initial condition. The class of differential equations is assumed to be approximated by well-posed truncated Taylor series expansions up to a certain order obtained about certain, in general nonperiodic, sampling points $t_{i} \in\left[t_{0}, t_{J}\right]$ for $i=0,1, \ldots, J$ of the solution. Two examples are provided.
\end{abstract}

\section{Introduction}

This paper investigates the errors of the solutions of nonlinear differential equations $\dot{y}(t)=f(y(t), t)$, where $f \in C^{(n+1)}\left(\mathbf{R}^{n} \times\right.$ $\left.\left(t_{0}, t_{T}\right) ; \mathbf{R}^{n}\right)$, provided they exist and are unique for each given admissible initial condition $y\left(t_{0}\right)=y_{0}$, with respect to the solutions of its approximate differential equations $\dot{x}(t)=$ $\sum_{k=0}^{\ell}\left(f^{(k)}\left(x\left(t_{i}\right), t_{i}\right) / k !\right)\left(x(t)-x\left(t_{i}\right)\right)^{k} ; x\left(t_{0}\right)=x_{0}$, for any given nonnegative integer $\ell \leq n$, obtained from truncated Taylor expansions of the solutions about certain sampling points $t_{i} \in\left[t_{0}, t_{J}\right]$ for $i=0,1, \ldots, J$. It is assumed that if a unique solution exists on some interval $\left[t_{0}, t_{J}\right]$ and that the choice of the sampling points is such that the intersample intervals [1-4] are subject to a certain maximum allowable upper-bound then the error of the solution in the whole interval $\left[t_{0}, t_{J}\right]$ satisfies a prescribed norm bound. Using the obtained results, the shadowing property [5-10] of the true solution with respect to the approximate one is investigated in the sense that "shadowing" initial conditions of the true solution exist, for each initial condition of the approximate differential equation, such that any approximated solution trajectory on the interval of interest is arbitrarily close to the true one under prescribed allowable maximum norms of the error between both the true solution and the approximate solutions. The problem is extended to the case when the approximated solution is perturbed either by a sequence of a certain allowable size at the sampling points or with perturbation functions of a certain size in norm about the whole considered interval. The main tool involved in the analysis is an "ad hoc" use of a known preparatory theorem to the celebrated Bernstein's theorem, [11], which gives an upper-bound for the maximum norm of the error in between both the true and the approximate solutions. The results are potentially extendable to functional equations involving nonlinearities and the presence of delays subject to mixed types of uncertainties [12-18]. On the other hand, different characterizations of oscillatory solutions and limit oscillatory solutions (limit cycles) have received important interest in the literature concerning different types of nonlinear dynamic continuous-time, discrete and hybrid systems, and differential equations [19-28]. The shadowing property is naturally relevant for the characterization of limit oscillations. Therefore, the formulation is applied in the second example to the characterization of limit cycles generated as solutions to Van der Pol's equation. 


\section{Calculation of the Exact Solution from Taylor Series Expansion}

Lemma 1. Assume that $f \in C^{(n+1)}\left((a, b) ; \mathbf{R}^{n}\right)$ and divide the real interval $(a, b)$ into $J$ subintervals with points $y_{n} \in[a, b]$ such that

$$
a \equiv y_{0}<y_{1}<y_{2}<\cdots<y_{J-1}<y_{J} \equiv b
$$

Then

$$
\begin{aligned}
f\left(y_{i+1}\right) & \\
= & f\left(y_{0}\right)+\int_{0}^{\bar{h}_{i}} f\left(y+y_{0}\right) d y \\
= & f\left(y_{0}\right)+\sum_{j=-1}^{i} \int_{\bar{h}_{j}}^{\bar{h}_{j+1}} f\left(y+y_{0}\right) d y \\
= & f\left(y_{0}\right)+\sum_{j=-1}^{i} \int_{0}^{h_{j}} f\left(y+y_{0}+\bar{h}_{j}\right) d y \\
= & f\left(y_{0}\right) \\
& +\sum_{j=-1}^{i} \sum_{k=0}^{n} \frac{f^{(k)}\left(c_{j}\right)}{k !} \int_{0}^{h_{j}}\left(y+y_{0}+\bar{h}_{j}-c_{j+1}\right)^{k} d y \\
& +\frac{1}{n !} \sum_{j=-1}^{i} \int_{0}^{h_{j}} \int_{c_{j+1}}^{y_{j+1}}\left(y+y_{0}+\bar{h}_{j}-t\right)^{n} f^{(n+1)}(t) d t d y
\end{aligned}
$$

where $h_{n}=y_{n+1}-y_{n}, \bar{h}_{n}=y_{n+1}-y_{0}=\sum_{i=0}^{n} h_{i}$ for $n=$ $0,1, \ldots, J-1$ with $\bar{h}_{-1}=0$, and

$$
\begin{aligned}
& f\left[y_{i+1}\left(\widetilde{h}_{i+1}\right)\right] \\
& =f\left(y_{0}\right) \\
& \quad+\sum_{j=-1}^{i} \sum_{k=0}^{n} \frac{f^{(k)}\left(c_{j}\right)}{k !} \int_{0}^{h_{j}}\left(y+y_{0}+\bar{h}_{j}-c_{j+1}\right)^{k} d y \\
& +\frac{1}{n !} \sum_{j=-1}^{i} \int_{0}^{h_{j}} \int_{c_{j+1}}^{y_{j+1}}\left(y+y_{0}+\bar{h}_{j}-t\right)^{n} f^{(n+1)}(t) d t d y \\
& \quad+\sum_{k=0}^{n} \frac{f^{(k)}\left(c_{i+1}\right)}{k !} \int_{0}^{\widetilde{h}_{i+1}}\left(y+y_{0}+\bar{h}_{i}+\widetilde{h}_{i+1}-c_{i+2}\right)^{k} d y \\
& \quad+\frac{1}{n !} \int_{0}^{\widetilde{h}_{i+1}} \int_{c_{j+1}}^{y_{j+1}}\left(y+y_{0}+\bar{h}_{i}+\widetilde{h}_{i+1}-t\right)^{n} f^{(n+1)}(t) d t d y ;
\end{aligned}
$$

$\forall y \in\left[y_{i}, y_{i+1}\right]$ and any real $c_{i} \in\left[y_{i}, y_{i+1}\right]$ for $i=0,1, \ldots, J-1$; $\forall \widetilde{h}_{i} \in\left[0, h_{i}\right]$ for $i=0,1, \ldots, J-2$.
Proof. It follows from a well-known preparatory theorem to Bernstein's theorem [5] that

$$
\begin{aligned}
f(y)= & \sum_{k=0}^{n} \frac{f^{(k)}\left(c_{i}\right)}{k !}\left(y-c_{i}\right)^{k} \\
& +\frac{1}{n !} \int_{c_{i}}^{y}(y-t)^{n} f^{(n+1)}(t) d t .
\end{aligned}
$$

Now, consider the nonlinear ordinary differential equation

$$
\dot{y}(t)=f(y(t), t) ; \quad y\left(t_{0}\right)=y_{0}
$$

in the real interval $\mathbf{R}^{n} \times\left[t_{0}, t_{J}\right]$ such that $f \in C^{(n+1)}\left(\mathbf{R}^{n} \times\right.$ $\left.\left(t_{0}, t_{J}\right) ; \mathbf{R}^{n}\right)$ is Lipschitz-continuous in $\left[y\left(t_{0}\right)-\theta_{0}, y\left(t_{0}\right)+\theta_{0}\right] \times$ $\left[t_{0}, t_{J}\right]$. The following result follows from Lemma 1 .

Theorem 2. The unique solution of $(5)$ in $\left[t_{0}, t_{J}\right]$ is given by

$$
\begin{array}{r}
y(t)=y\left(t_{0}\right) \\
+\sum_{j=0}^{i-2} \int_{0}^{h_{j}}\left[\sum_{k=0}^{\ell} \frac{f^{(k)}\left(y\left(t_{j}\right), t_{j}\right)}{k !}\left(y\left(\tau+t_{j}\right)-y\left(t_{j}\right)\right)^{k}\right. \\
+\frac{1}{\ell !} \int_{0}^{\tau}\left(y\left(\sigma+t_{j}\right)-y\left(t_{j}\right)\right)^{\ell} \\
+\int_{t_{i-1}}^{t}\left[\sum_{k=0}^{\ell} \frac{f^{(k)}\left(y\left(t_{j}\right), t_{j}\right)}{k !}\left(y\left(\tau+t_{i-1}\right)-y\left(t_{i-1}\right)\right)^{k}\right. \\
\left.+\frac{1}{\ell !} \int_{0}^{\tau}\left(y\left(\sigma+t_{j-1}\right), \sigma+t_{j}\right) d \sigma\right] d \tau \\
\left.\times f^{(\ell+1)}\left(y\left(\sigma+t_{i-1}\right), \sigma+t_{i-1}\right) d \sigma\right] d \tau ;
\end{array}
$$

$\forall t \in\left[t_{i-1}, t_{i}\right] ; \forall i \in \bar{J}=\{1,2, \ldots, J\}$ and $\forall \ell\left(\in \mathbf{Z}_{0+}\right) \leq n$, where $t_{i} \in\left[t_{0}, t_{J}\right]$ are any arbitrary strictly ordered points such that $t_{0}<t_{1}<t_{2}<\cdots<t_{J-1}<t_{J}$ with $h_{i}=t_{i+1}-t_{i}$ for $i=0,1, \ldots, J-1$.

Proof. Note that $f \in C^{(n+1)}\left(\mathbf{R}^{n} \times\left(t_{0}, t_{J}\right) ; \mathbf{R}^{n}\right)$ is Lipschitzcontinuous in $\left[y\left(t_{0}\right)-\theta_{0}, y\left(t_{0}\right)+\theta_{0}\right] \times\left[t_{0}, t_{J}\right]$ so that the solution $y(t)$ on $\left[t_{0}, t_{J}\right]$ is unique, provided that $t_{J}=t_{J}\left(\theta_{0}, t_{0}\right)$ for the given $t_{0} \in \mathbf{R}$ and some $\theta_{0} \in \mathbf{R}^{n}$ is such that $y(t) \in$ $\left[y\left(t_{0}\right)-\theta_{0}, y\left(t_{0}\right)+\theta_{0}\right] ; \forall t \in\left[t_{0}, t_{J}\right]$ and $t_{J} \in\left(t_{0}, t_{\bar{J}}\right]$ since $f:\left[y\left(t_{0}\right)-\theta_{0}, y\left(t_{0}\right)+\theta_{0}\right] \times\left[t_{0}, t_{J}\right] \rightarrow \mathbf{R}^{n}$ is local Lipschitzcontinuous as a result. Such a unique solution is given by

$$
y(t)=y_{a}+\int_{a}^{t} f(y(\tau), \tau) d \tau ; \quad \forall t \in\left[t_{0}, t_{J}\right] .
$$


Take any set of $J$ strictly ordered points $t_{n} \in\left[t_{0}, t_{J}\right]$ satisfying $t_{0}<t_{1}<t_{2}<\cdots<t_{J-1}<t_{J}$ with $h_{i}=t_{i+1}-t_{i}$ for $i=$ $0,1, \ldots, J-1$, so that

$$
\begin{aligned}
y(t) & =y\left(t_{i-1}\right)+\int_{t_{i-1}}^{t} f(y(\tau), \tau) d \tau \\
& =y\left(t_{0}\right)+\sum_{j=0}^{i-2} \int_{t_{j}}^{t_{j+1}} f(y(\tau), \tau) d \tau+\int_{t_{i-1}}^{t} f(y(\tau), \tau) d \tau
\end{aligned}
$$

$\forall t \in\left[t_{i-1}, t_{i}\right] ; \forall i \in \bar{J}=\{1,2, \ldots, J\}$, with $y\left(t_{0}\right)$, so that, by choosing the real $c_{i} \in\left[t_{i-1}, t_{i}\right]$ as $c_{i}=t_{i}$ for $i=0,1, \ldots, J-1$, one gets from (3) in the proof of Lemma 1 into (4):

$$
\begin{aligned}
& y(t)= y\left(t_{0}\right) \\
&+ \sum_{j=0}^{i-2} \int_{0}^{h_{j}}\left[\sum_{k=0}^{n} \frac{f^{(k)}\left(y\left(t_{j}\right), t_{j}\right)}{k !}\left(y\left(\tau+t_{j}\right)-y\left(t_{j}\right)\right)^{k}\right. \\
&+\frac{1}{n !} \int_{0}^{\tau}\left(y\left(\sigma+t_{j}\right)-y\left(t_{j}\right)\right)^{n} \\
&+\int_{0}^{t-t_{i-1}}\left[\sum_{k=0}^{n} \frac{f^{(k)}\left(y\left(t_{i-1}\right), t_{i-1}\right)}{k !}\right.\left.\times f^{(n+1)}\left(y\left(\sigma+t_{j}\right), \sigma+t_{j}\right) d \sigma\right] d \tau \\
& \times\left(y\left(\tau+t_{i-1}\right)-y\left(t_{i-1}\right)\right)^{k} \\
&+\frac{1}{n !} \int_{0}^{\tau}\left(y\left(\sigma+t_{i-1}\right)-y\left(t_{i-1}\right)\right)^{n} \\
&\left.\times f^{(n+1)}\left(y\left(\sigma+t_{i-1}\right), \sigma+t_{i-1}\right) d \sigma\right] d \tau ;
\end{aligned}
$$

$\forall t \in\left[t_{i-1}, t_{i}\right] ; \forall i \in \bar{J}=\{1,2, \ldots, J\}$. Note that, since $f \in$ $C^{(n+1)}\left(\mathbf{R}^{n} \times\left(t_{0}, t_{J}\right) ; \mathbf{R}^{n}\right)$, then $f \in C^{(\ell+1)}\left(\mathbf{R}^{n} \times\left(t_{0}, t_{J}\right) ; \mathbf{R}^{n}\right)$ for any nonnegative integer $\ell \leq n$. Thus, we obtain the result from a similar expression of (9) by replacing $n$ by $\ell(\leq n)$ while truncating the Taylor series expansion by its $(\ell+1)$ th term.

A consequence of Theorem 2 by using the same technique of the solution construction is as follows.

Corollary 3. Consider the nonlinear ordinary differential equation (5) with initial condition $y\left(t_{0}\right)$ on the real interval $\mathbf{R}^{n} \times \mathbf{R}_{0+}$, with initial conditions $y^{(j)}\left(t_{0}\right)$ for $j=0,1, \ldots, n-1$, such that $f \in C^{(n+1)}\left(\mathbf{R}^{n} \times\left(t_{0}, t_{J}\right) ; \mathbf{R}^{n}\right)$ is Lipschitz-continuous in $\left[y\left(t_{0}\right)-\theta_{0}, y\left(t_{0}\right)+\theta_{0}\right] \times\left[t_{0}, t_{J}\right]$ for some $\theta_{0} \in \mathbf{R}^{n}$, and consider also its $\ell$ th order truncation

$$
\begin{array}{r}
\dot{x}(t)=\sum_{k=0}^{\ell} \frac{f^{(k)}\left(x\left(t_{i}\right), t_{i}\right)}{k !}\left(x(t)-x\left(t_{i}\right)\right)^{k} ; \\
x\left(t_{0}\right)=x_{0}
\end{array}
$$

such that $f^{(k)}(y(t), t)$ are bounded in $\left[y\left(t_{0}\right)-\theta, y\left(t_{0}\right)+\theta\right] \times$ $\left[t_{0}, t_{J}\right]$ for $k=0,1, \ldots, \ell+1$ for some nonnegative integer $\ell \leq$ $n$ and some $\theta \in \bar{B}\left(\theta_{0}, R\right)$, where $\bar{B}(\theta, R)=\left\{z \in \mathbf{R}^{n}: \| z-\right.$ $\left.\theta_{0} \| \leq R\right\}$ for some positive real $R$ with $x^{(j)}\left(t_{0}\right)=y^{(j)}\left(t_{0}\right)$ for $j=0,1, \ldots, \ell+1$.

Since $f^{(k)}(y(t), t)$ are bounded in $\left[y\left(t_{0}\right)-\theta, y\left(t_{0}\right)+\theta\right] \times$ $\left[t_{0}, \bar{t}_{J}\right]$ for $k=0,1, \ldots, \ell-1$, then the right-hand-side of (10) is Lipschitz-continuous in $\left[y\left(t_{0}\right)-\theta, y\left(t_{0}\right)+\theta\right] \times\left[t_{0}, t_{J}\right] \subseteq$ $\left[y\left(t_{0}\right)-\theta, y\left(t_{0}\right)+\theta\right] \times\left[t_{0}, t_{J}\right]$. Therefore, the unique solution of the truncated differential equation $(10)$ in $[a, b]$ is

$x(t)=x\left(t_{0}\right)$

$$
\begin{gathered}
+\sum_{k=0}^{\ell}\left\{\sum_{j=0}^{i-2} \int_{0}^{h_{j}}\left[\frac{f^{(k)}\left(x\left(t_{j}\right), t_{j}\right)}{k !}\left(x\left(\tau+t_{j}\right)-x\left(t_{j}\right)\right)^{k}\right] d \tau\right. \\
+\int_{0}^{t-t_{i-1}}\left[\frac{f^{(k)}\left(x\left(t_{i-1}\right), t_{i-1}\right)}{k !}\right. \\
\left.\left.\times\left(x\left(\sigma+t_{i-1}\right)-x\left(t_{i-1}\right)\right)^{k}\right] d \tau\right\} ;
\end{gathered}
$$

$\forall t \in\left[t_{i-1}, t_{i}\right] ; \forall i \in \bar{J}=\{1,2, \ldots, J\}, \forall \ell\left(\in \mathbf{Z}_{0+}\right) \leq n$, where $t_{i} \in\left[t_{0}, t_{J}\right]$ are arbitrary strictly ordered points such that $t_{0}<$ $t_{1}<t_{2}<\cdots<t_{J-1}<t_{J}$ with $h_{i}=t_{i+1}-t_{i}$ for $i=0,1, \ldots, J-1$. The error in between the exact solution of (10) and that of its truncated form (5) is

$$
\begin{aligned}
& e(t)=y(t)-x(t) \\
& =\sum_{j=0}^{i-2} \sum_{k=0}^{\ell} \int_{0}^{h_{j}}\left(\frac{f^{(k)}\left(y\left(t_{j}\right), t_{j}\right)}{k !}\left(y\left(\tau+t_{j}\right)-y\left(t_{j}\right)\right)^{k}\right. \\
& -\frac{f^{(k)}\left(x\left(t_{j}\right), t_{j}\right)}{k !} \\
& \left.\times\left(x\left(\tau+t_{j}\right)-x\left(t_{j}\right)\right)^{k}\right) d \tau \\
& +\frac{1}{\ell !} \sum_{j=0}^{i-2} \int_{0}^{h_{j}} \int_{0}^{\tau}\left(y\left(\sigma+t_{j}\right)-y\left(t_{j}\right)\right)^{\ell} \\
& \times f^{(\ell+1)}\left(y\left(\sigma+t_{j}\right), \sigma+t_{j}\right) d \sigma d \tau \\
& +\sum_{k=0}^{\ell} \int_{0}^{t-t_{i-1}}\left(\frac{f^{(k)}\left(y\left(t_{i-1}\right), t_{i-1}\right)}{k !}\right. \\
& \times\left(y\left(\tau+t_{i-1}\right)-y\left(t_{i-1}\right)\right)^{k} \\
& -\frac{f^{(k)}\left(x\left(t_{i-1}\right), t_{i-1}\right)}{k !} \\
& \left.\times\left(x\left(\tau+t_{i-1}\right)-x\left(t_{i-1}\right)\right)^{k}\right) d \tau
\end{aligned}
$$




$$
\begin{aligned}
+\frac{1}{\ell !} \int_{0}^{t-t_{i-1}} & \int_{0}^{\tau}\left(y\left(\sigma+t_{i-1}\right)-y\left(t_{i-1}\right)\right)^{\ell} \\
& \times f^{(\ell+1)}\left(y\left(\sigma+t_{i-1}\right), \sigma+t_{i-1}\right) d \sigma d \tau
\end{aligned}
$$

$\forall t \in\left[t_{i-1}, t_{i}\right] ; \forall i \in \bar{J}=\{1,2, \ldots, J\}$ and $\forall \ell\left(\in \mathbf{Z}_{0+}\right) \leq n$.

Proof. Property (i) follows directly Theorem 2 applied to the truncated differential equation (10) leading to the solution (11) in $\left[t_{0}, t_{J}\right]$. Property (ii) follows from (6) and (11).

Now, a preparatory result follows to be then used to guarantee sufficiency-type errors results in between the true and the approximate solutions in the interval $[a, b]$.

Lemma 4. Assume that the following hypothesis holds.

(A1) $f(y(t), t)$ and its first $(\ell+1)$ derivatives are uniformly bounded from above on a bounded subset of their existence domain with the specific boundedness constraint:

$$
\begin{aligned}
& \sup _{y(t) \in\left[y\left(t_{0}\right)-\theta, y\left(t_{J}\right)+\theta\right], t \in\left[t_{0}, t_{J}\right]}\|f(y(t), t)\| \\
& \leq K \sup _{y(t) \in\left[y\left(t_{0}\right)-\theta, y\left(t_{J}\right)+\theta\right], t \in\left[t_{0}, t_{J}\right]}\|y(t)\|+K_{1}, \\
& \sup _{y(t) \in\left[y\left(t_{0}\right)-\theta, y\left(t_{J}\right)+\theta\right], t \in\left[t_{0}, t_{J}\right]}\left\|f^{(j)}(y(t), t)\right\| \\
& \leq K \sup _{y(t) \in\left[y\left(t_{0}\right)-\theta, y\left(t_{J}\right)+\theta\right], t \in\left[t_{0}, t_{J}\right]}\left\|f^{(j-1)}(y(t), t)\right\|+K_{1},
\end{aligned}
$$

for $j=0,1, \ldots, \ell+1$ and some $K, K_{1} \in \mathbf{R}_{0+}$ with $K<1$ if $K_{1} \in \mathbf{R}_{+}$. Then, the following properties hold.

(i) Assume that the intersample intervals $h_{i}=t_{i+1}-t_{i}$ for $i=0,1, \ldots, J-1$ fulfill the constraint

$$
\begin{aligned}
h_{i} & \leq h \\
& \leq \min \left(a_{i}, \frac{1-\rho_{x} / 2}{\Lambda_{x}\left(1-\rho_{x} / 2\right)^{\ell}}, \frac{\rho_{x}}{2 \Lambda_{x}\left(1-\rho_{x} / 2\right)^{\ell+1}\left(J-1+\rho_{x} / 2\right)}\right)
\end{aligned}
$$

for $i=0,1, \ldots, J-1$ and any given real constant $\rho_{x} \in(0,2)$, where

$$
\begin{aligned}
& \Lambda_{x}= \sum_{k=0}^{\ell} \frac{K^{k}}{k !}\left(\sup _{y(t) \in\left[y\left(t_{0}\right)-\theta, y\left(t_{J}\right)+\theta\right], t \in\left[t_{0}, t_{J}\right]}\|f(y(t), t)\|\right) \\
&+\frac{K_{1}\left(1-K^{\ell+1}\right)}{1-K} \\
& a_{i}:=\min \arg \left(t\left(\in \mathbf{R}_{+}\right)>t_{i}:\left\|x(t)-x\left(t_{i}\right)\right\| \leq \frac{\rho_{x}}{2}\right) \\
& \forall t \in\left[t_{i}, t_{i+1}\right), \quad i=0,1, \ldots, J-1 .
\end{aligned}
$$

Then, the approximated solution fulfills $\sup _{t \in\left[t_{0}, t_{J}\right]} \| x(t)-$ $x\left(t_{0}\right) \| \leq \rho_{x} / 2$ provided that

$$
t_{1}=\min \arg \left(t\left(\in \mathbf{R}_{+}\right)>t_{0}:\left\|x(t)-x\left(t_{0}\right)\right\| \leq \frac{\rho_{x}}{2}\right) .
$$

(ii) Assume that the intersample intervals $h_{i}=t_{i+1}-t_{i}$ for $i=0,1, \ldots, J-1$ fulfill the constraint

$h_{i} \leq h$

$$
\leq \min \left(b_{i}, \frac{1-\rho / 2}{\Lambda(1-\rho / 2)^{\ell}}, \frac{\rho}{2 \Lambda(1-\rho / 2)^{\ell+1}(J-1+\rho / 2)}\right)
$$

for $i=0,1, \ldots, J-1$ and any given real constant $\rho \in(0,1)$, where

$$
\begin{gathered}
\Lambda=\sum_{k=0}^{\ell+1} \frac{K^{k}}{k !}\left[\left(\sup _{y(t) \in\left[y\left(t_{0}\right)-\theta, y\left(t_{J}\right)+\theta\right], t \in\left[t_{0}, t_{J}\right]}\|f(y(t), t)\|\right)\right. \\
\left.+\frac{K_{1}\left(1-K^{k}\right)}{1-K}\right] \\
b_{i}:=\min \arg \left(t>t_{i}:\left\|y(t)-y\left(t_{i}\right)\right\| \leq \frac{\rho}{2}\right) \\
\forall t \in\left[t_{i}, t_{i+1}\right), \quad i=0,1, \ldots, J-1
\end{gathered}
$$

Then, the true solution fulfills $\sup _{t \in\left[t_{0}, t_{j}\right]}\left\|y(t)-y\left(t_{0}\right)\right\| \leq \rho / 2$ provided that

$$
t_{1}=\min \arg \left(t\left(\in \mathbf{R}_{+}\right)>t_{0}:\left\|y(t)-y\left(t_{0}\right)\right\| \leq \frac{\rho}{2}\right) .
$$

(iii) If $\rho_{x}=\rho \in(0,1)$ and, furthermore,

$h_{i} \leq h$

$$
\begin{array}{r}
\leq \min \left(c_{i}, \frac{1-\rho / 2}{\Lambda(1-\rho / 2)^{\ell}}, \frac{\rho}{2 \Lambda(1-\rho / 2)^{\ell+1}(J-1+\rho / 2)}\right) \\
i=0,1, \ldots, J-1,
\end{array}
$$

$$
\begin{aligned}
c_{i}:=\min \arg ( & t>t_{i}: \max \left(\left\|y(t)-y\left(t_{i}\right)\right\|,\left\|x(t)-x\left(t_{i}\right)\right\|\right) \\
& \left.\leq \frac{\rho}{2}\right) ; \quad \forall t \in\left[t_{i}, t_{i+1}\right), i=0,1, \ldots, J-1,
\end{aligned}
$$$$
t_{1}=\min \arg \left(t\left(\epsilon \mathbf{R}_{+}\right)\right.
$$

$$
\begin{aligned}
& >t_{0}: \max \left(\left\|y(t)-y\left(t_{0}\right)\right\|,\left\|x(t)-x\left(t_{0}\right)\right\|\right) \\
& \left.\leq \frac{\rho}{2}\right)
\end{aligned}
$$


then the true, the approximated and the error solution fulfill

$$
\begin{aligned}
& \sup _{t \in\left[t_{0}, t_{J}\right]}\left\|y(t)-y\left(t_{0}\right)\right\| \leq \frac{\rho}{2}, \\
& \sup _{t \in\left[t_{0}, t_{J}\right]}\left\|x(t)-x\left(t_{0}\right)\right\| \leq \frac{\rho}{2},
\end{aligned}
$$

and the error in between them, $e(t)=y(t)-x(t)$, fulfills

$$
\sup _{t \in\left[t_{0}, t_{j}\right]}\left\|e(t)-e\left(t_{0}\right)\right\| \leq \rho
$$

Proof. Proceeding recursively, one gets from Assumption A1 that

$$
\begin{aligned}
& \sup _{y(t) \in\left[y\left(t_{0}\right)-\theta, y\left(t_{J}\right)+\theta\right], t \in\left[t_{0}, t_{J}\right]}\left\|f^{(j)}(y(t), t)\right\| \\
& \leq \sup _{y(t) \in\left[y\left(t_{0}\right)-\theta, y\left(t_{J}\right)+\theta\right], t \in\left[t_{0}, t_{J}\right]}\left\|f^{(j-1)}(y(t), t)\right\|+K_{1} \\
& \leq K_{y(t) \in\left[y\left(t_{0}\right)-\theta, y\left(t_{J}\right)+\theta\right], t \in\left[t_{0}, t_{J}\right]}^{2}\left\|f^{(j-1)}(y(t), t)\right\| \\
& \quad+K_{1}(1+K) \\
& \leq K^{k} F_{0}+K_{1}\left(\sum_{i=0}^{k-1} K^{i}\right) \leq K^{k} F_{0}+\frac{K_{1}\left(1-K^{k}\right)}{1-K} \\
& \leq F_{0}+\frac{K_{1}}{1-K}
\end{aligned}
$$

if $K<1$ and $K_{1} \neq 0$, and

$$
\sup _{y(t) \in\left[y\left(t_{0}\right)-\theta, y\left(t_{J}\right)+\theta\right], t \in\left[t_{0}, t_{J}\right]}\left\|f^{(k)}(y(t), t)\right\| \leq K^{k} F_{0}
$$

if $K_{1}=0$, where

$$
\begin{aligned}
F_{0} & =\sup _{y(t) \in\left[y\left(t_{0}\right)-\theta, y\left(t_{J}\right)+\theta\right], t \in\left[t_{0}, t_{J}\right]}\left\|f^{(0)}(y(t), t)\right\| \\
& =\sup _{y(t) \in\left[y\left(t_{0}\right)-\theta, y\left(t_{J}\right)+\theta\right], t \in\left[t_{0}, t_{J}\right]}\|f(y(t), t)\|<+\infty .
\end{aligned}
$$

Case (a). If $K<1$ and $K_{1} \neq 0$ proceed by complete induction by assuming that $\sup _{t \in\left[t_{0}, t_{i}\right]}\left\|x(t)-x\left(t_{0}\right)\right\| \leq \rho_{x} / 2$ since the condition $\left(t\left(\in \mathbf{R}_{+}\right)>t_{i}:\left\|x(t)-x\left(t_{i}\right)\right\| \leq \rho_{x} / 2\right)$ guarantees that $\sup _{t \in\left[t_{0}, t_{1}\right]}\left\|x(t)-x\left(t_{0}\right)\right\| \leq \rho_{x} / 2$. Thus, one gets from (11) that

$$
\begin{aligned}
\| x( & t)-x\left(t_{i}\right) \| \\
\leq & \sum_{k=0}^{\ell} \sum_{j=0}^{i} \frac{h_{j}}{k !}\left(K^{k} F_{0}+\frac{K_{1}\left(1-K^{k}\right)}{1-K}\right)\left(\frac{\rho_{x}}{2}\right)^{k} \\
= & \sum_{k=0}^{\ell} \sum_{j=0}^{i-1} \frac{h_{j}}{k !}\left(K^{k} F_{0}+\frac{K_{1}\left(1-K^{k}\right)}{1-K}\right)\left(\frac{\rho_{x}}{2}\right)^{k} \\
& +\sum_{k=0}^{\ell} \frac{h_{i}}{k !}\left(K^{k} F_{0}+\frac{K_{1}\left(1-K^{k}\right)}{1-K}\right)\left\|x(t)-x\left(t_{i}\right)\right\|^{k} \\
\leq & \sum_{k=0}^{\ell} \sum_{j=0}^{i-1} \frac{h_{j}}{k !}\left(K^{k} F_{0}+\frac{K_{1}\left(1-K^{k}\right)}{1-K}\right)\left(\frac{\rho_{x}}{2}\right)^{k} \\
& +\sum_{k=0}^{\ell} \frac{h_{i}}{k !}\left(K^{k} F_{0}+\frac{K_{1}\left(1-K^{k}\right)}{1-K}\right)\left\|x(t)-x\left(t_{i}\right)\right\|^{k} \\
= & \Lambda_{x}\left(\sum_{k=0}^{\ell}\left(\frac{\rho_{x}}{2}\right)^{k}\right)\left(\sum_{j=0}^{i-1} h_{j}\right) \\
& +h_{i} \Lambda\left[\sum_{k=0}^{\ell}\left(\left\|x(t)-x\left(t_{i}\right)\right\|^{k}\right)\right] \\
\leq & i h \Lambda_{x}\left(\sum_{k=0}^{\ell}\left(\frac{\rho_{x}}{2}\right)^{k}\right)\left\|x(t)-x\left(t_{i}\right)\right\| ; \\
& \\
& \\
&
\end{aligned}
$$

where $\Lambda_{x}=\sum_{k=0}^{\ell}(1 / k !)\left(K^{k} F_{0}+\left(K_{1}\left(1-K^{k}\right) /(1-K)\right)\right)$ and $h \geq \max _{0 \leq i \leq J-1} h_{i}$, with $h_{i}=t_{i+1}-t_{i}$, for $i=0,1, \ldots, J-1$, so that

$$
\begin{aligned}
{[1-} & \left.h_{i} \Lambda_{x}\left(\sum_{k=0}^{\ell}\left(\left\|x(t)-x\left(t_{i}\right)\right\|^{k-1}\right)\right)\right] \\
& \times\left\|x(t)-x\left(t_{i}\right)\right\| \leq i h \Lambda_{x}\left(\sum_{k=0}^{\ell}\left(\frac{\rho_{x}}{2}\right)^{k}\right) \\
= & \frac{i h \Lambda_{x}\left(1-\left(\rho_{x} / 2\right)^{\ell+1}\right)}{1-\rho_{x} / 2}
\end{aligned}
$$

or

$$
\begin{aligned}
& \left\|x(t)-x\left(t_{i}\right)\right\| \\
& \leq \frac{i h \Lambda_{x}\left(1-\left(\rho_{x} / 2\right)^{\ell+1}\right)}{\left[1-h_{i} \Lambda_{x}\left(\sum_{k=0}^{\ell}\left(\left\|x(t)-x\left(t_{i}\right)\right\|^{k-1}\right)\right)\right]\left(1-\rho_{x} / 2\right)} \\
& \quad \leq \frac{i h \Lambda_{x}\left(1-\left(\rho_{x} / 2\right)^{\ell+1}\right)}{\left[1-\left(\left(h_{i} \Lambda_{x}\left(1-\left(\rho_{x} / 2\right)^{\ell}\right)\right) /\left(1-\rho_{x} / 2\right)\right)\right]\left(1-\rho_{x} / 2\right)} \\
& \quad \leq \frac{\rho_{x}}{2}
\end{aligned}
$$


provided that $0<\rho_{x}<2$, and $1>h_{i} \Lambda_{x}\left(\sum_{k=0}^{\ell}(\| x(t)-\right.$ $\left.\left.x\left(t_{i}\right) \|^{k-1}\right)\right)$ which is guaranteed if $h_{i}<\min \left(a_{i},(1-\right.$ $\left.\left.\rho_{x} / 2\right) /\left(\Lambda_{x}\left(1-\rho_{x} / 2\right)^{\ell}\right)\right)$ holds with $a_{i}$ for $i=0,1, \ldots, J-1$, defined in (16), provided that $\left\|x(t)-x\left(t_{j}\right)\right\| \leq \rho_{x} / 2 ; \forall t \in$ $\left[t_{j}, t_{j+1}\right)$ for $j(\leq i)=0,1, \ldots, i-1$, and then (33) and $h_{j}<$ $\left(\left(1-\rho_{x} / 2\right) /\left(\Lambda_{x}\left(1-\rho_{x} / 2\right)^{\ell}\right)\right)$ for $j=0,1, \ldots, i-1$ are jointly guaranteed for the given $i=0,1, \ldots, J-1$ if

$h_{i}$

$$
<\min \left(\frac{1-\rho_{x} / 2}{\Lambda_{x}\left(1-\rho_{x} / 2\right)^{\ell}}, \frac{\rho_{x}}{2 \Lambda_{x}\left(1-\rho_{x} / 2\right)^{\ell+1}\left(J-1+\rho_{x} / 2\right)}\right)
$$

provided that $\left\|x(t)-x\left(t_{j}\right)\right\| \leq \rho_{x} / 2$ for $t \in\left[t_{j}, t_{j+1}\right)$ for $j=$ $0,1, \ldots, i-1$, the last condition being identical to

$$
t_{i+1} \leq a_{i}:=\min \arg \left(t>t_{i}:\left\|x(t)-x\left(t_{i}\right)\right\| \leq \frac{\rho_{x}}{2}\right) .
$$

The above two conditions (34)-(35) reduce to (14). Then, one gets from complete induction from (31), if (14) holds, the following.

$\sup _{t \in\left[t_{0}, t_{i}\right]}\left\|x(t)-x\left(t_{i}\right)\right\| \leq \rho_{x} / 2 \Rightarrow \sup _{t \in\left[t_{0}, t_{i+1}\right)} \| x(t)-$ $x\left(t_{i}\right) \| \leq \rho_{x} / 2$ and one gets also by continuity extension,

$\sup _{t \in\left[t_{0}, t\right]}\left\|x(t)-x\left(t_{0}\right)\right\| \leq \rho_{x} / 2 ; \forall t \in\left[t_{0}, t_{J}\right]$. Hence, we got the result for Case (a).

Case (b). If $K_{1}=0$ then

$$
\begin{aligned}
\left\|x(t)-x\left(t_{i}\right)\right\| \\
\leq i h \Lambda_{x 0}\left(\sum_{k=0}^{\ell}\left(\frac{\rho_{x}}{2}\right)^{k}\right) \\
\quad+h_{i} \Lambda_{x 0}\left(\sum_{k=0}^{\ell}\left(\left\|x(t)-x\left(t_{i}\right)\right\|^{k-1}\right)\right)\left\|x(t)-x\left(t_{i}\right)\right\| ;
\end{aligned}
$$

$\forall t \in\left[t_{i}, t_{i+1}\right)$, where $\Lambda_{x 0}=\sum_{k=0}^{\ell}\left(\left(K^{k} F_{0}\right) /(k !)\right) \leq \Lambda_{x}$, so that $\left(1-h \Lambda_{x 0}\right)\left\|x(t)-x\left(t_{i}\right)\right\| \leq i h \Lambda_{x 0} E$ for $i=0,1, \ldots, J$ and, thus, one gets the following.

$\left\|x(t)-x\left(t_{i}\right)\right\| \leq\left(\left(i h \Lambda_{x 0} E\right) /\left(1-h \Lambda_{x 0}\right)\right) \leq \rho_{x} / 2 ; \forall t \in$ $\left[t_{i}, t_{i+1}\right)$ for $i=0,1, \ldots, J$ and one gets from complete induction the same conclusion $\sup _{t \in\left[t_{0}, t\right]}\left\|x(t)-x\left(t_{0}\right)\right\| \leq \rho_{x} / 2$; $\forall t \in\left[t_{0}, t_{J}\right)$ as in Case (a) provided that (14) holds. Then, (14) guarantees Property (i) for both Cases (a) and (b). Then, Property (i) has been proven.

Property (ii) is proven "mutatis-mutandis" by noting that $\Lambda \geq \Lambda_{x}$ from (15) and (19) and noting also that $a_{i}$ in (16) is replaced with $b_{i}$ in (20) so that the admissible intersample interval satisfying the constraint (14) is replaced by such an interval satisfying the constraint (18). Finally, Property (iii) follows from Properties (i)-(ii) by equalizing $\rho_{x}$ and $\rho$ to take a maximum value less than $1 / 2$.

The following comments address the fact that it is not necessary to deal with the solution of the true differential equation (5) to calculate upper-bounds of the solution and error in Lemma 4.
Remark 5. Note that one gets by direct integration from (5) that

$$
\begin{aligned}
& \|y(t)\| \\
& \quad \leq \sup _{t_{0} \leq \tau \leq t_{J}}\|y(\tau)\| \\
& \leq\left\|y\left(t_{0}\right)\right\|+\left(t_{J}-t_{0}\right) K \sup _{t_{0} \leq \tau \leq t_{J}}\|y(\tau)\|
\end{aligned}
$$

leading to

$$
\sup _{t_{0} \leq t \leq t_{J}}\|y(t)\| \leq \frac{K\left(t_{J}-t_{0}\right)}{1-K\left(t_{J}-t_{0}\right)}\left\|y\left(t_{0}\right)\right\| \quad \text { if } \sum_{i=0}^{J-1} h_{i}<\frac{1}{K} .
$$

Thus, (25)-(26) might be guaranteed with $\sup _{t_{0} \leq t \leq t_{I}} \| y(t)-$ $y\left(t_{0}\right)\left\|\leq\left(1 /\left(1-K\left(t_{J}-t_{0}\right)\right)\right)\right\| y\left(t_{0}\right) \| \leq(\rho / 2)$ if $\left\|y\left(t_{0}\right)\right\| \leq$ $(\rho / 2)\left(1-K\left(t_{J}-t_{0}\right)\right)<(\rho / 2)$. Thus, there is no need to compute the solution of the true differential equation (5) and $\sup _{t_{i} \leq t<t_{i+1}}\left\|y(t)-y\left(t_{0}\right)\right\| \leq(\rho / 2)$ for $i=0,1, \ldots, J-1$ in (20) and (23) if $\left\|y\left(t_{0}\right)\right\| \leq(\rho / 2)\left(1-K\left(t_{J}-t_{0}\right)\right)$.

One gets directly from Lemma 4 the subsequent result.

Theorem 6. Assume the conditions (13a) and (13b) and (22)(24) of Lemma 4(iii). Then

$$
\begin{gathered}
\max \left(\max _{0 \leq i \leq J-1}\left\|e\left(t_{i+1}\right)-e\left(t_{i}\right)\right\|,\left\|e(t)-e\left(t_{0}\right)\right\|\right) \leq \rho<1 ; \\
\max _{t \in\left[t_{0}, t_{J}\right]}\|e(t)\| \leq\left\|e\left(t_{0}\right)\right\|+\rho ;
\end{gathered}
$$

$\forall t \in\left[t_{0}, t_{J}\right]$, and

$$
\begin{gathered}
\max _{0 \leq i \leq J-1}\left\|e(t)-e\left(t_{i}\right)\right\| \leq \rho ; \\
\forall t \in\left[t_{i}, t_{i+1}\right] \text { for } i=0,1, \ldots, J-1
\end{gathered}
$$

for $i=0,1, \ldots, J-1$.

\section{Orbits of the Exact Solution, \\ Pseudo-Orbits of the Approximated Solution, and the Shadowing Property}

Now, consider a perturbed solution (11) of the approximated differential equation (10) associated with a perturbation $x\left(t_{i}\right)=x\left(t_{i}^{-}\right)+g\left(t_{i}\right)$ with $\left\{g\left(t_{i}\right)\right\} \subset \mathbf{R}^{n}$ at $t=t_{i}$ fulfilling $\left\|g\left(t_{i}\right)\right\| \leq \bar{g}_{i} \leq \bar{g}$ for some given $\bar{g} \in \mathbf{R}, \forall i \in \bar{J}$. Note that a perturbation at the initial $t=t_{0}$ is considered by choosing $x\left(t_{0}\right)=y\left(t_{0}\right)+g_{0}$ for some nonzero $g_{0}=g\left(t_{0}\right) \epsilon$ $\mathbf{R}$. The perturbed solution can be generated, in particular, from impulsive controls of amplitudes $g\left(t_{i}\right)$ at the sequence 
$\left\{t_{i}: i \in \bar{J}\right\}$. The exact and approximate solutions (6) and (11) in $\left[t_{0}, t_{J}\right]$, provided that they exist, are

$$
\begin{array}{r}
y(t)=y\left(t_{i}\right) \\
+\sum_{k=0}^{\ell} \int_{0}^{t-t_{i}} \frac{f^{(k)}\left(y\left(t_{i}\right), t_{i}\right)}{k !}\left(y\left(\tau+t_{i}\right)-y\left(t_{i}\right)\right)^{k} d \tau \\
+\frac{1}{\ell !} \int_{0}^{t-t_{i}} \int_{0}^{\tau}\left(y\left(\sigma+t_{j}\right)-y\left(t_{j}\right)\right)^{\ell} \\
\times f^{(\ell+1)}\left(y\left(\sigma+t_{j}\right), \sigma+t_{j}\right) d \sigma d \tau ; \\
\forall t \in\left[t_{i}, t_{i+1}\right], i=0,1, \ldots, J-1,
\end{array}
$$

$$
\begin{aligned}
x(t)= & x\left(t_{i}\right) \\
& +\sum_{k=0}^{\ell} \int_{0}^{t-t_{i}} \frac{f^{(k)}\left(x\left(t_{i}\right), t_{i}\right)}{k !}\left(x\left(\tau+t_{i}\right)-x\left(t_{i}\right)\right)^{k} d \tau \\
+ & U\left(t-t_{i+1}\right) g\left(t_{i+1}\right) ; \\
& \forall t \in\left[t_{i}, t_{i+1}\right], i=0,1, \ldots, J-1,
\end{aligned}
$$

where $U(t)$ is the Heaviside function. The error between the exact and perturbed approximated solutions becomes

$$
\begin{gathered}
e(t)=e\left(t_{i}\right) \\
+\sum_{k=0}^{\ell} \int_{0}^{t-t_{i}}\left(\frac{f^{(k)}\left(y\left(t_{i}\right), t_{i}\right)}{k !}\left(y\left(\tau+t_{i}\right)-y\left(t_{i}\right)\right)^{k}\right. \\
-\frac{f^{(k)}\left(x\left(t_{i}\right), t_{i}\right)}{k !} \\
\left.\times\left(x\left(\tau+t_{i}\right)-x\left(t_{i}\right)\right)^{k}\right) d \tau \\
+\frac{1}{\ell !} \int_{0}^{t-t_{i}} \int_{0}^{\tau}\left(y\left(\sigma+t_{j}\right)-y\left(t_{j}\right)\right)^{\ell} \\
\times f^{(\ell+1)}\left(y\left(\sigma+t_{j}\right), \sigma+t_{j}\right) d \sigma d \tau \\
-U\left(t-t_{i+1}\right) g\left(t_{i+1}\right) ;
\end{gathered}
$$

$\forall t \in\left[t_{i}, t_{i+1}\right] ; i=0,1, \ldots, J-1$. Now, one gets from (25)-(26) of Lemma 4:

$$
\begin{aligned}
& \left\|e(t)-e\left(t_{i}\right)\right\| \\
& \leq \sum_{k=0}^{\ell} \frac{2^{k+1}}{k !}\left(t-t_{i}\right) M_{i k}\left(\frac{\rho}{2}\right)^{k} \\
& \quad+\frac{1}{\ell !}\left(t-t_{i}\right)^{2} 2^{\ell} M_{i, \ell+1}\left(\frac{\rho}{2}\right)^{\ell}+\bar{g}_{i} ; \quad \forall t \in\left[t_{i}, t_{i+1}\right],
\end{aligned}
$$

where

$$
\begin{aligned}
M_{i k} & =\sup _{y(t) \in\left[y\left(t_{i}\right)-\theta, y\left(t_{i}\right)+\theta\right], t \in\left[t_{i}, t_{i+1}\right]}\left\|f^{(j)}(y(t), t)\right\| \\
& \leq K^{k} F_{0}+\frac{K_{1}\left(1-K^{k}\right)}{1-K} \\
& \leq \frac{K^{k+1} \rho}{2}+\frac{K_{1}\left(1-K^{k}\right)}{1-K} \leq \frac{\rho}{2}+\frac{K_{1}}{1-K}
\end{aligned}
$$

from (27) and one gets after using the triangle inequality,

$$
\begin{aligned}
\left\|e(t)-e\left(t_{i}\right)\right\| \\
\leq \sum_{j=i}^{m}\left(\sum_{k=0}^{\ell} \frac{2^{k+1}}{k !}\left(t-t_{j}\right)\left[\frac{K^{k+1} \rho}{2}+\frac{K_{1}\left(1-K^{k}\right)}{1-K}\right]\left(\frac{\rho}{2}\right)^{k}\right. \\
+\frac{1}{\ell !}\left(t-t_{j}\right)^{2} 2^{\ell} \\
\left.\quad \times\left[\frac{K^{\ell+2} \rho}{2}+\frac{K_{1}\left(1-K^{\ell+1}\right)}{1-K}\right]\left(\frac{\rho}{2}\right)^{\ell}+\bar{g}_{j}\right) ;
\end{aligned}
$$

$\forall t \in\left[t_{i+m}, t_{i+m+1}\right]$ for $m=0,1, \ldots, J-i-1 ; i=0,1, \ldots, J-1$. One obtains easily from (46), either by complete induction or via recursive calculation, that

$$
\begin{aligned}
& \left\|e(t)-e\left(t_{i}\right)\right\| \\
& \begin{aligned}
\leq \sum_{j=0}^{J-1}\left(\sum_{k=0}^{\ell} \frac{2^{k+1}}{k !}\left(t-t_{j}\right)\left[\frac{K^{k+1} \rho}{2}+\frac{K_{1}\left(1-K^{k}\right)}{1-K}\right]\left(\frac{\rho}{2}\right)^{k}\right. \\
+\frac{1}{\ell !}\left(t-t_{j}\right)^{2} 2^{\ell} \\
\left.\times\left[\frac{K^{\ell+2} \rho}{2}+\frac{K_{1}\left(1-K^{\ell+1}\right)}{1-K}\right]\left(\frac{\rho}{2}\right)^{\ell}+\bar{g}_{j}\right)
\end{aligned}
\end{aligned}
$$

$$
\begin{aligned}
\leq J h\left(\sum_{k=0}^{\ell} \frac{2^{k+1}}{k !}\left[\frac{K^{k+1} \rho}{2}+\frac{K_{1}\left(1-K^{k}\right)}{1-K}\right]\left(\frac{\rho}{2}\right)^{k}\right. \\
+\frac{J h}{\ell !} 2^{\ell} \\
\left.\quad \times\left[\frac{K^{\ell+2} \rho}{2}+\frac{K_{1}\left(1-K^{\ell+1}\right)}{1-K}\right]\left(\frac{\rho}{2}\right)^{\ell}\right)+J \bar{g},
\end{aligned}
$$


$\|e(t)\| \leq\left\|e\left(t_{0}\right)\right\|$

$$
\begin{aligned}
+\sum_{j=0}^{J-1}\left(t-t_{j}\right)\left(\sum_{k=0}^{\ell}\right. & \frac{2^{k+1}}{k !}\left[\frac{K^{k+1} \rho}{2}+\frac{K_{1}\left(1-K^{k}\right)}{1-K}\right]\left(\frac{\rho}{2}\right)^{k} \\
& +\frac{1}{\ell !}\left(t-t_{j}\right) 2^{\ell} \\
& \times\left[\frac{K^{\ell+2} \rho}{2}+\frac{K_{1}\left(1-K^{\ell+1}\right)}{1-K}\right] \\
& \left.\times\left(\frac{\rho}{2}\right)^{\ell}+\bar{g}_{j}\right)
\end{aligned}
$$

$\leq\left\|e\left(t_{0}\right)\right\|$

$$
\begin{aligned}
+J h\left(\sum_{k=0}^{\ell} \frac{2^{k+1}}{k !}\left[\frac{K^{k+1} \rho}{2}+\frac{K_{1}\left(1-K^{k}\right)}{1-K}\right]\left(\frac{\rho}{2}\right)^{k}\right. \\
\left.+\frac{J h}{\ell !} 2^{\ell}\left[\frac{K^{\ell+2} \rho}{2}+\frac{K_{1}\left(1-K^{\ell+1}\right)}{1-K}\right]\left(\frac{\rho}{2}\right)^{\ell}\right)
\end{aligned}
$$

$+J \bar{g}$

$\forall t \in\left[t_{0}, t_{0}+\sum_{i=0}^{J-1} h_{i}\right]\left(\subseteq\left[t_{0}, t_{0}+J h\right]\right)$ with $h_{i}=t_{i+1}-t_{i}$ for $i=0,1, \ldots, J-1$ and any given nonnegative integer $\ell \leq n$. The following result follows directly from the above equations and Theorem 6.

Theorem 7. Consider an approximated perturbed solution (42) under a forcing perturbation sequence $\left\{g\left(t_{i}\right)\right\} \subset \mathbf{R}^{n}$ at $t=t_{i}$ fulfilling $\left\|g\left(t_{i}\right)\right\| \leq \bar{g}_{i} \leq \bar{g} \geq\left\|e\left(t_{0}\right)\right\|$ for $i=1,2, \ldots$ and some $\bar{g} \in \mathbf{R}_{+}$. Then, there are numbers $h \in \mathbf{R}_{+}, J=J(h) \in \mathbf{Z}_{+}$, $\varepsilon_{1}=\varepsilon_{1}(h, \bar{g}) \in \mathbf{R}_{+}$, and $\varepsilon=\varepsilon\left(\varepsilon_{1},\left\|e\left(t_{0}\right)\right\|\right)$ such that

$$
\begin{gathered}
\max \left(\max _{0 \leq i \leq J-1}\left\|e\left(t_{i+1}\right)-e\left(t_{i}\right)\right\|, \max _{t \in \mathbf{R}_{0+}}\left\|e(t)-e\left(t_{0}\right)\right\|\right) \leq \varepsilon_{1} ; \\
\max _{t \in \mathbf{R}_{0+}}\|e(t)\| \leq \varepsilon
\end{gathered}
$$

on $\left[t_{0}, t_{J}\right]$ for any strictly ordered sequence of $(J+1)$ nonnegative real numbers $\left\{t_{i}: i=0,1, \ldots, J\right\}$, subject to the constraints

$$
\begin{gathered}
t_{J}=t_{0}+\sum_{i=0}^{J-1} h_{i}, \\
h_{i}=t_{i+1}-t_{i} \leq h, \\
i=0,1, \ldots, J-1
\end{gathered}
$$

Proof. Note that fixing $\sum_{i=0}^{J-1} h_{i}=t_{J}-t_{0} \leq J h$, with $h=$ $\max _{0 \leq i \leq J-1}\left(t_{i+1}-t_{i}\right)$, and the use of (46), (47a), and (47b) leads to

$$
\begin{aligned}
& \left\|e(t)-e\left(t_{i}\right)\right\| \\
& \leq \sum_{i=0}^{J-1}\left(h _ { i } \left(\sum_{k=0}^{\ell} \frac{2^{k+1}}{k !}\left[\frac{K^{k+1} \rho}{2}+\frac{K_{1}\left(1-K^{k}\right)}{1-K}\right]\left(\frac{\rho}{2}\right)^{k}\right.\right. \\
& +\frac{1}{\ell !} h 2^{\ell} \\
& \left.\left.\times\left[\frac{K^{\ell+2} \rho}{2}+\frac{K_{1}\left(1-K^{\ell+1}\right)}{1-K}\right]\left(\frac{\rho}{2}\right)^{\ell}\right)+\bar{g}_{i}\right) \\
& \leq \varepsilon_{1}=\rho+\sum_{i=0}^{J-1} \bar{g}_{i} \\
& \left\|e(t)-e\left(t_{i}\right)\right\| \\
& \leq J h\left(\sum_{k=0}^{\ell} \frac{2^{k+1}}{k !}\left[\frac{K^{k+1} \rho}{2}+\frac{K_{1}\left(1-K^{k}\right)}{1-K}\right]\left(\frac{\rho}{2}\right)^{k}\right. \\
& \left.+\frac{1}{\ell !} h 2^{\ell}\left[\frac{K^{\ell+2} \rho}{2}+\frac{K_{1}\left(1-K^{\ell+1}\right)}{1-K}\right]\left(\frac{\rho}{2}\right)^{\ell}\right)
\end{aligned}
$$$$
+J \bar{g} \leq \bar{\varepsilon}_{1}=\bar{\rho}+J \bar{g}
$$

(50b)

$\forall t \in\left[t_{0}, t_{J}\right] ; i=0,1, \ldots, J-1$ by using Lemma 4 and Theorem 6 for any given prefixed $\rho \in \mathbf{R}_{+}$. The result then follows since $\bar{g}_{0}=\left\|e\left(t_{0}\right)\right\| \leq \bar{g}$ and either

$$
\begin{gathered}
\varepsilon=\varepsilon_{1}+\left\|e\left(t_{0}\right)\right\|, \quad \varepsilon_{1}=\rho+\sum_{i=0}^{J-1} \bar{g}_{i}, \\
\sum_{i=0}^{J-1} h_{i}\left(\sum_{k=0}^{\ell} \frac{2^{k+1}}{k !}\left[\frac{K^{k+1} \rho}{2}+\frac{K_{1}\left(1-K^{k}\right)}{1-K}\right]\left(\frac{\rho}{2}\right)^{k}\right. \\
\left.+\frac{h}{\ell !} 2^{\ell}\left[\frac{K^{\ell+2} \rho}{2}+\frac{K_{1}\left(1-K^{\ell+1}\right)}{1-K}\right]\left(\frac{\rho}{2}\right)^{\ell}\right) \leq \rho
\end{gathered}
$$

or

$$
\begin{gathered}
\varepsilon=\bar{\varepsilon}_{1}+\left\|e\left(t_{0}\right)\right\|, \quad \bar{\varepsilon}_{1}=\bar{\rho}+J \bar{g} \\
h\left(\sum_{k=0}^{\ell} \frac{2^{k+1}}{k !}\left[\frac{K^{k+1} \rho}{2}+\frac{K_{1}\left(1-K^{k}\right)}{1-K}\right]\left(\frac{\rho}{2}\right)^{k}\right. \\
\left.+\frac{1}{\ell !} h 2^{\ell}\left[\frac{K^{\ell+2} \rho}{2}+\frac{K_{1}\left(1-K^{\ell+1}\right)}{1-K}\right]\left(\frac{\rho}{2}\right)^{\ell}\right) \leq \bar{\rho} ;
\end{gathered}
$$

and the result has been proven. 
The following result extends Theorem 7 with results of Theorem 6 for the case when both the exact and approximated differential equations are subject to a piecewisecontinuous bounded disturbance which might be dependent on the solution and also can have finite step discontinuities in the sequence $\left\{t_{i}: i=0,1, \ldots, J\right\}$.

Theorem 8. Consider the forced versions of the differential equations (5) and (10):

$$
\begin{gathered}
\dot{y}(t)=f(y(t), t)+g(\tau, y(\tau)), \quad y\left(t_{0}\right)=y_{0}, \quad \\
\dot{x}(t)=\sum_{k=0}^{\ell} \frac{f^{(k)}\left(x\left(t_{i}\right), t_{i}\right)}{k !}\left(x(t)-x\left(t_{i}\right)\right)^{k}+g(\tau, x(\tau)), \\
x\left(t_{0}\right)=x_{0}
\end{gathered}
$$

under the additive forcing perturbation $g \in C^{(n+1)}\left(\mathbf{R}^{n} \times\right.$ $\left.\left(t_{0}, t_{J}\right) ; \mathbf{R}^{n}\right)$ satisfying Assumption (A2) of Lemma 4 fulfilling $g(y(t), t)=\lambda(t) y(t)$ and $g(x(t), t)=\lambda(t) x(t)+U\left(t-t_{i+1}\right) g_{i+1}$; $\forall t \in\left[t_{i}, t_{i+1}\right]$ with $\left\|g_{i+1}\right\| \leq \bar{g}$ for $i=0,1, \ldots, J-1$ and some $\bar{g} \in \mathbf{R}_{+}$and $\lambda:\left[t_{0}, t_{J}\right] \rightarrow \mathbf{R}^{n}$ being a bounded piecewisecontinuous function. Then, there are numbers $h \in \mathbf{R}_{+}, J=$ $J(h) \in \mathbf{Z}_{+}, \varepsilon_{1}=\varepsilon_{1}(h, \bar{g}) \in \mathbf{R}_{+}$and $\varepsilon=\varepsilon\left(\varepsilon_{1},\left\|e\left(t_{0}\right)\right\|\right)$ such that

$$
\begin{gathered}
\max \left(\max _{0 \leq i \leq J-1}\left\|e\left(t_{i+1}\right)-e\left(t_{i}\right)\right\|, \max _{t \in \mathbf{R}_{0+}}\left\|e(t)-e\left(t_{0}\right)\right\|\right) \leq \varepsilon_{1}, \\
\max _{t \in \mathbf{R}_{0_{+}}}\|e(t)\| \leq \rho
\end{gathered}
$$

on $\left[t_{0}, t_{J}\right]$ for a strictly ordered finite set of $(J+1)$ nonnegative real numbers $\left\{t_{i}: i=0,1, \ldots, J\right\}$, subject to the constraints $t_{J}=t_{0}+\sum_{i=0}^{J-1} h_{i}, h_{i}=t_{i+1}-t_{i} \leq h ; i=0,1, \ldots, J-1$, the constraints (22)-(24) subject to (18), and either

$$
\begin{gathered}
\sum_{i=0}^{J-1} h_{i} \lambda_{i}<1, \\
\sum_{i=0}^{J-1} \bar{g}_{i}<\infty, \quad \bar{g} \geq \frac{\sum_{i=0}^{J-1} h_{i} \lambda_{i}}{1-\sum_{i=0}^{J-1} h_{i} \lambda_{i}}\left\|e\left(t_{0}\right)\right\|,
\end{gathered}
$$

or

$J h \lambda<1$

$$
J \bar{g}<\infty, \quad \bar{g} \geq \frac{J h \lambda}{1-J h \lambda}\left\|e\left(t_{0}\right)\right\| .
$$

Proof. Fix $\sum_{i=0}^{J-1} h_{i}=t_{J}-t_{0} \leq J h$, with $h=\max _{0 \leq i \leq J-1}\left(t_{i+1}-t_{i}\right)$. Equations (53)-(54) have the following solutions:

$$
\begin{aligned}
& y(t)=y\left(t_{i}\right) \\
& +\sum_{k=0}^{\ell} \int_{0}^{t-t_{i}}\left(\frac{f^{(k)}\left(y\left(t_{i}\right), t_{i}\right)}{k !}\left(y\left(\tau+t_{i}\right)-y\left(t_{i}\right)\right)^{k}\right. \\
& +\frac{1}{\ell !} \int_{0}^{\tau}\left(y\left(\sigma+t_{j}\right)-y\left(t_{j}\right)\right)^{\ell} \\
& \quad \times f^{(\ell+1)}\left(y\left(\sigma+t_{j}\right), \sigma+t_{j}\right) d \sigma \\
& +g(y(\tau), \tau)) d \tau, \\
& x(t)=x\left(t_{i}\right) \quad \\
& +\int_{0}^{t-t_{i}}\left(\sum_{k=0}^{\ell} \frac{f^{(k)}\left(x\left(t_{i}\right), t_{i}\right)}{k !}\left(x\left(\tau+t_{i}\right)-x\left(t_{i}\right)\right)^{k}\right. \\
& +g(x(\tau), \tau)) d \tau \\
& +U\left(t-t_{i+1}\right) g_{i+1} ;
\end{aligned}
$$

$\forall t \in\left[t_{i}, t_{i+1}\right], i=0,1, \ldots, J-1$. Note that

$$
g(y(t), t)-g(x(t), t)=\lambda(t)(y(t)-x(t))=\lambda(t) e(t) .
$$

Thus, the error between both of them becomes

$$
\begin{aligned}
& e(t)=e\left(t_{i}\right) \\
& +\sum_{k=0}^{\ell} \int_{0}^{t-t_{i}}\left(\frac{f^{(k)}\left(y\left(t_{i}\right), t_{i}\right)}{k !}\left(y\left(\tau+t_{i}\right)-y\left(t_{i}\right)\right)^{k}\right. \\
& -\frac{f^{(k)}\left(x\left(t_{i}\right), t_{i}\right)}{k !}\left(x\left(\tau+t_{i}\right)-x\left(t_{i}\right)\right)^{k} \\
& +g(e(\tau), \tau)) d \tau \\
& +\frac{1}{\ell !} \int_{0}^{t-t_{i}} \int_{0}^{\tau}\left(y\left(\sigma+t_{j}\right)-y\left(t_{j}\right)\right)^{\ell} \\
& \times f^{(\ell+1)}\left(y\left(\sigma+t_{j}\right), \sigma+t_{j}\right) d \sigma d \tau \\
& -U\left(t-t_{i+1}\right) g\left(x\left(t_{i+1}\right), t_{i+1}\right) ;
\end{aligned}
$$


$\forall t \in\left[t_{i}, t_{i+1}\right]$. Then, (45) leads to

$$
\begin{aligned}
& \|e(t)\| \\
& \leq\left\|e\left(t_{i}\right)\right\|+\left(t-t_{i}\right) \\
& \quad \times\left(\sum_{k=0}^{\ell} \frac{2^{k+1}}{k !}\left[\frac{K^{k+1} \rho}{2}+\frac{K_{1}\left(1-K^{k}\right)}{1-K}\right]\left(\frac{\rho}{2}\right)^{k}\right. \\
& +\frac{1}{\ell !}\left(t-t_{i}\right) 2^{\ell} \\
& \left.\quad \times\left[\frac{K^{\ell+2} \rho}{2}+\frac{K_{1}\left(1-K^{\ell+1}\right)}{1-K}\right]\left(\frac{\rho}{2}\right)^{\ell}+g_{i+1}\right) ;
\end{aligned}
$$

$\forall t \in\left[t_{i}, t_{i+1}\right], i=0,1, \ldots, J-1$. Then

$$
\begin{aligned}
& \sup _{t_{i} \leq t \leq t_{i+1}}(\|e(t)\|) \\
& \leq\left\|e\left(t_{i}\right)\right\|+\left(t-t_{i}\right) \\
& \times\left(\sum_{k=0}^{\ell} \frac{2^{k+1}}{k !}\left[\frac{K^{k+1} \rho}{2}+\frac{K_{1}\left(1-K^{k}\right)}{1-K}\right]\left(\frac{\rho}{2}\right)^{k}\right. \\
& +\frac{1}{\ell !}\left(t-t_{i}\right) 2^{\ell} \\
& \left.\quad \times\left[\frac{K^{\ell+2} \rho}{2}+\frac{K_{1}\left(1-K^{\ell+1}\right)}{1-K}\right]\left(\frac{\rho}{2}\right)^{\ell}+g_{i+1}\right) \\
& +h_{i} \lambda_{i} \sup _{t_{i} \leq \tau \leq t_{i+1}}(\|e(\tau)\|)+\bar{g}
\end{aligned}
$$

so that, since $1>h_{i} \lambda_{i}$, where $\lambda_{i}=\max _{t_{i} \leq \tau \leq t_{i+1}}|\lambda(\tau)|$ for $i=$ $0,1, \ldots, J-1$, one gets

$$
\begin{aligned}
& \sup _{t_{i} \leq t \leq t_{i+1}}(\|e(t)\|) \\
& \leq \frac{1}{1-h_{i} \lambda_{i}} \\
& \quad \times\left(\left\|e\left(t_{i}\right)\right\|\right. \\
& +\left(\sum_{k=0}^{\ell} \frac{2^{k+1}}{k !} h_{i}\left[\frac{K^{k+1} \rho}{2}+\frac{K_{1}\left(1-K^{k}\right)}{1-K}\right]\left(\frac{\rho}{2}\right)^{k}\right. \\
& \quad+\frac{1}{\ell !}\left(t-t_{i}\right) 2^{\ell} \\
& \left.\left.\quad \times\left[\frac{K^{\ell+2} \rho}{2}+\frac{K_{1}\left(1-K^{\ell+1}\right)}{1-K}\right]\left(\frac{\rho}{2}\right)^{\ell}+g_{i+1}\right)\right)
\end{aligned}
$$

which implies

$$
\begin{aligned}
& \left|\sup _{t_{i} \leq t \leq t_{i+1}}\left(\|e(t)\|-\left\|e\left(t_{i}\right)\right\|\right)\right| \\
& \leq \frac{h_{i} \lambda_{i}}{1-h_{i} \lambda_{i}}\left\|e\left(t_{i}\right)\right\|+\frac{1}{1-h_{i} \lambda_{i}} \\
& \times\left(\sum_{k=0}^{\ell} \frac{2^{k+1}}{k !} h_{i}\left[\frac{K^{k+1} \rho}{2}+\frac{K_{1}\left(1-K^{k}\right)}{1-K}\right]\left(\frac{\rho}{2}\right)^{k}\right. \\
& +\frac{1}{\ell !} h_{i}^{2} 2^{\ell} \\
& \left.\times\left[\frac{K^{\ell+2} \rho}{2}+\frac{K_{1}\left(1-K^{\ell+1}\right)}{1-K}\right]\left(\frac{\rho}{2}\right)^{\ell}+g_{i+1}\right) .
\end{aligned}
$$

If $\sum_{i=0}^{J-1} h_{i} \lambda_{i}<1$, we also get (66)-(67) below from (65) as well as (68)-(69) if, in addition, $\operatorname{Jh} \lambda<1$ :

$$
\begin{aligned}
& \sup _{t_{0} \leq t \leq t_{J}}(\|e(t)\|) \\
& \leq \frac{1}{1-\sum_{i=0}^{J-1} h_{i} \lambda_{i}} \\
& \quad \times\left(\left\|e\left(t_{0}\right)\right\|\right. \\
& +\sum_{i=0}^{J-1} h_{i}\left(\sum_{k=0}^{\ell} \frac{2^{k+1}}{k !}\left[\frac{K^{k+1} \rho}{2}+\frac{K_{1}\left(1-K^{k}\right)}{1-K}\right]\left(\frac{\rho}{2}\right)^{k}\right. \\
& \quad+\frac{1}{\ell !} h_{i} 2^{\ell} \\
& \left.\quad \times\left[\frac{K^{\ell+2} \rho}{2}+\frac{K_{1}\left(1-K^{\ell+1}\right)}{1-K}\right]\left(\frac{\rho}{2}\right)^{\ell}\right) \\
& \left.+\sum_{i=0}^{J-1} \bar{g}_{i}\right),
\end{aligned}
$$

$$
\begin{aligned}
& \left|\sup _{t_{0} \leq t \leq t_{J}}\left(\|e(t)\|-\left\|e\left(t_{0}\right)\right\|\right)\right| \\
& \leq \frac{\sum_{i=0}^{J-1} h_{i} \lambda_{i}}{1-\sum_{i=0}^{J-1} h_{i} \lambda_{i}} \\
& \times\left(\left\|e\left(t_{0}\right)\right\|\right. \\
& \quad+\sum_{i=0}^{J-1} h_{i}\left(\sum_{k=0}^{\ell} \frac{2^{k+1}}{k !}\left[\frac{K^{k+1} \rho}{2}+\frac{K_{1}\left(1-K^{k}\right)}{1-K}\right]\left(\frac{\rho}{2}\right)^{k}\right. \\
& \quad+\frac{1}{\ell !} h_{i} 2^{\ell} \\
& \left.\quad \times\left[\frac{K^{\ell+2} \rho}{2}+\frac{K_{1}\left(1-K^{\ell+1}\right)}{1-K}\right]\left(\frac{\rho}{2}\right)^{\ell}\right) \\
& \left.+\sum_{i=0}^{J-1} \bar{g}_{i}\right)
\end{aligned}
$$




$$
\begin{aligned}
& \sup _{t_{0} \leq t \leq t_{J}}(\|e(t)\|) \\
& \leq \frac{1}{1-J h \lambda} \\
& \times\left(\left\|e\left(t_{0}\right)\right\|\right. \\
& +J h\left(\sum_{k=0}^{\ell} \frac{2^{k+1}}{k !}\left[\frac{K^{k+1} \rho}{2}+\frac{K_{1}\left(1-K^{k}\right)}{1-K}\right]\left(\frac{\rho}{2}\right)^{k}\right. \\
& +\frac{1}{\ell !} h 2^{\ell} \\
& \left.\times\left[\frac{K^{\ell+2} \rho}{2}+\frac{K_{1}\left(1-K^{\ell+1}\right)}{1-K}\right]\left(\frac{\rho}{2}\right)^{\ell}\right) \\
& +J \bar{g}) \\
& \left|\sup _{t_{0} \leq t \leq t_{J}}\left(\|e(t)\|-\left\|e\left(t_{0}\right)\right\|\right)\right| \\
& \leq \frac{J h \lambda}{1-J h \lambda} \\
& \times J h\left(\sum_{k=0}^{\ell} \frac{2^{k+1}}{k !}\left[\frac{K^{k+1} \rho}{2}+\frac{K_{1}\left(1-K^{k}\right)}{1-K}\right]\left(\frac{\rho}{2}\right)^{k}\right. \\
& +\frac{1}{\ell !} h 2^{\ell} \\
& \left.\times\left[\frac{K^{\ell+2} \rho}{2}+\frac{K_{1}\left(1-K^{\ell+1}\right)}{1-K}\right]\left(\frac{\rho}{2}\right)^{\ell}\right)
\end{aligned}
$$

where $\lambda=\max _{0 \leq t \leq t_{I}}|\lambda(t)|=\max _{0 \leq i \leq J-1} \max _{t_{i} \leq \tau \leq t_{i+1}}|\lambda(\tau)|=$ $\max _{0 \leq i \leq J-1} \lambda_{i}$. Property (i) follows from (65)-(66) by defining $\varepsilon_{0}, \varepsilon_{1}$, and $\varepsilon$ as in (51) since $\bar{g} \geq$ $\max \left(\max _{0 \leq i \leq J-1}\left\|g_{i+1}\right\|,\left(\left(\sum_{i=0}^{J-1} h_{i} \lambda_{i}\right) /\left(1-\sum_{i=0}^{J-1} h_{i} \lambda_{i}\right)\right)\left\|e\left(t_{0}\right)\right\|\right)$ and Property (ii) follows from (67)-(68) by defining $\rho, \varepsilon_{1}$, and $\varepsilon$ as in (52) since $\bar{g} \geq \max \left(\max _{0 \leq i \leq J-1}\left\|g_{i+1}\right\|,((J h \lambda) /(1-\right.$ $\left.J h \lambda))\left\|e\left(t_{0}\right)\right\|\right)$. Thus, the result has been proven.

Now, three definitions are given concerning the so-called pseudo-orbits, as a counterpart to the true sampled trajectory solution, or orbit, of finite size $J$ of the approximate solutions and their perturbed version within the given classes of perturbations. The related concepts are relevant for then quantifying the maximum errors among the real and approximated solutions and parallel issues concerning their counterparts under perturbations of the studied types. More specifically refer to the following.

Definition 9. A sampling sequence $\widehat{t}_{J}=\left\{t_{i}: i=0,1, \ldots, J\right\}$ of strictly ordered sampling points with $h_{i}=t_{i+1}-t_{i} \leq h$; $i=0,1, \ldots, J-1$ is said to be in the class $C_{J h}=\left\{t_{i} \in \widehat{t}_{J}\right.$ : $\left.t_{i+1}-t_{i} \leq h ; i=0,1, \ldots, J-1\right\}$.

Note from Definition 9 that $h \leq h^{\prime} \Rightarrow C_{J h} \subseteq C_{J h^{\prime}}$ and that $\widehat{t}_{J} \equiv\left\{t_{i}: i=0,1, \ldots, J-1\right\} \subset C_{J h} \Rightarrow t_{J}-t_{0} \leq J h$.

Definition 10. A sequence $\widehat{x}_{J}=\left\{x\left(t_{i}\right): i=0,1, \ldots, J-1\right\}$ of $J$ samples of the solution of an approximate differential equation (10) is a $\delta$-pseudo $J$-orbit of sampling sequence $\widehat{t}_{J}$ for some $\delta \in \mathbf{R}_{+}$and is denoted by $O\left(\widehat{x}_{J}, \Gamma, \delta\right)$ if $\max _{t \in\left[t_{0}, t_{J}\right]}\|e(t)\| \leq \delta$.

If the integer $J$ and the real $t_{J}$ are infinite, the corresponding trajectory solutions are referred to as complete pseudoorbits and orbits. The solution of the true differential equation (5) is a $J$-orbit of sampling sequence $\hat{t}_{J}$. The continuous approximate (resp., true) solution for $\left[t_{0}, t_{J}\right]$ is the $\delta$-pseudo $J$-orbit (resp., $J$-orbit) of sampling sequence $\widehat{t}_{J}$. The perturbed solutions under the forcing perturbations of Theorems 7 and 8 are denoted in a similar way leading to the corresponding perturbed pseudo-orbits.

Definition 11. The set of all the $\delta$-pseudo $J$-orbits $O\left(\widehat{x}_{J}, \Gamma, \delta\right)$ with $\max _{t \in\left[t_{0}, t_{J}\right]}\|e(t)\| \leq \delta$, for some $\delta \in \mathbf{R}_{+}$, obtained for any sampling sequence $\widehat{t}_{J} \in C_{J h}$, is said to be the class $C O\left(C_{J h}, \delta\right)$ of $\delta$-pseudo $J$-orbits of sampling sequence $\widehat{t}_{J}$.

The mapping which generates the true solution sequences, for given initial conditions and sampling sequence, has the shadowing property if there is an arbitrarily close orbit for any given $\delta$-pseudo-orbit $O\left(\widehat{x}_{J}, \Gamma, \delta\right)$ in the following precise sense.

Definition 12. The set $\widehat{Y}_{J}$ of true solution sequences $\widehat{y}_{J}=$ $\left\{y\left(t_{i}\right): t_{i} \in \widehat{t}_{J}, i=0,1, \ldots, J-1\right\}$ of sampling sequence $\widehat{t}_{J}$ possesses the shadowing property on the corresponding set of approximate solutions if, for each given $\delta \in \mathbf{R}_{+}$, there is some $y_{0}=y_{0}(\delta)$ for which a $O\left(\hat{x}_{J}, \Gamma, \delta\right)$ exists. It is said that $y_{0}=y_{0}(\delta)$ shadows $O\left(\widehat{x}_{J}, \Gamma, \delta\right)$.

The subsequent result establishes that if the set of true solution sequences has the shadowing property then the class $C O\left(C_{J h}, \delta\right)$ of $\delta$-pseudo $J$-orbits of sampling sequence $\widehat{t}_{J}$ is nonempty for any $\delta \in \mathbf{R}_{+}$.

Proposition 13. If the set $\widehat{Y}_{J}$ of true solution sequences of sampling sequence $\widehat{t}_{J}$ possesses the shadowing property then $\mathrm{CO}\left(C_{J h}, \delta\right)$ is nonempty for any $\delta \in \mathbf{R}_{+}$.

Note that $C O\left(C_{J h}, \rho\right)=\bigcup_{\Gamma \in C_{J h}} O\left(\widehat{x}_{J}, \Gamma, \rho\right)$ and note also that $C O\left(C_{J h}, \rho\right) \subseteq C O\left(C_{J h^{\prime}}, \rho\right)$ for any $h^{\prime} \geq h$. The subsequent result relies on Theorem 7 and Definition 11 for a class of pseudo-orbits $C O\left(C_{J h}, \rho\right)$ defined by a sampling sequence class $C_{J h}$. In fact, the characterization becomes global for all approximated solutions on a finite interval $\left[t_{0}, t_{J}\right]$ for sampling intervals $h_{i}=t_{i+1}-t_{i} \leq h ; i=0,1, \ldots, J-$ 1 and initial conditions subject to a maximum allowable deviation with respect to the initial condition of the true solution provided that the approximate solution exists in a global (rather than local) definition domain. 
The so-called shadowing properties, [5-9], of the true solutions with respect to the approximated ones rely on the physical meaning that for sets of appropriate initial conditions, the true solution is arbitrarily close to its approximate version on a certain interval $\left[t_{0}, t_{J}\right]$ where both solutions exist and are unique. Based on Theorems 6, 7, and 8, the shadowing properties of the true solution to the approximated solution, those ones being the nominal one or the perturbed ones under the class of perturbations of Theorems 7 and 8, are now discussed. It is seen that the shadowing properties at sampling points under Theorems 6,7 , and 8 guarantee the corresponding properties in $\left[t_{0}, t_{J}\right]$.

The shadowing properties of true solutions of pseudoorbits for constrained sampling sequences according to the constraints of Theorem 6 are addressed in the subsequent result.

Proposition 14. Consider the true and approximated solutions associated with the differential equations (5) and (10) satisfying the hypotheses and conditions of Theorem 6. Then, such a set of solutions lies in the class $\mathrm{CO}\left(C_{J h}, \varepsilon\right)$ of $\varepsilon$-pseudo $J$-orbits of sampling sequence $\widehat{t}_{J}=\widehat{t}_{J}(\rho)$ for $\rho \leq \varepsilon$, subject to one of the constraints (13a), (13b), (22)-(24) (Lemma 4, Theorem 6), belonging to a sampling sequence class $C_{J h}$ for any $\rho, \varepsilon \in \mathbf{R}_{+}$with arbitrary $\rho \leq \varepsilon$ and any given $\varepsilon$. Also, there is a $y_{0}=y_{0}(\varepsilon)$ which shadows each $\mathrm{O}\left(\widehat{x}_{J}, \Gamma, \varepsilon\right) \in \mathrm{CO}\left(C_{J h}, \varepsilon\right)$ for each given $\varepsilon \in \mathbf{R}_{+}$and $\rho \leq \varepsilon$.

Proof. One gets from Theorem 6 that

$$
\max _{t \in\left[t_{0}, t_{J}\right]}\|e(t)\| \leq \varepsilon=\left\|e\left(t_{0}\right)\right\|+\rho
$$

for an initial condition $y\left(t_{0}\right)$ of the true differential equation fulfilling $\left|\left\|y\left(t_{0}\right)\right\|-\left\|x\left(t_{0}\right)\right\|\right| \leq\left\|e\left(t_{0}\right)\right\|$ and any given real constants $\varepsilon \geq \rho>0$. This defines families of initial conditions $y_{0}=y_{0}(\varepsilon)$ of the true differential equation which shadow each $O\left(\widehat{x}_{J}, \Gamma, \varepsilon\right) \in C O\left(C_{J h}, \varepsilon\right)$ for each given $\varepsilon \in \mathbf{R}_{+}$and $\rho \leq \varepsilon$. For any given $\varepsilon \in \mathbf{R}_{+}$, it suffices to take $0<\rho \leq \varepsilon$ to zero in (22)-(24) of Lemma 4 and (39)-(40) in Theorem 6 to fix an admissible sampling sequence $\widehat{t}_{J}=\widehat{t}_{J}(\varepsilon)$ and then to get the result.

The perturbed approximated differential equations referred to in Theorems 8 and 7, which is a particular case of Theorem 8 for $g(x(t), t)$ being zero for $t \notin \widehat{t}_{J}$, that is for nonsampling points, are analyzed in the subsequent result which generalizes Proposition 14.

Theorem 15. Consider the true and approximated solutions (58) and (59) associated with the differential equations satisfying the hypotheses and conditions of Theorem 8. Then, such a set of solutions lies in the class $\mathrm{CO}\left(C_{J h}, \varepsilon\right)$ of $\varepsilon$ pseudo J-orbits of sampling sequence $\widehat{t}_{J}=\widehat{t}_{J}(\rho)$, for some $\rho>0$, subject to one of the constraints (13a), (13b), (22)-(24), and (39)-(40) (Lemma 4, Theorem 6) and to either (56a) or (56b) (Theorem 8) with $\rho \leq \varepsilon-$ $\sum_{i=0}^{J-1} \bar{g}_{i}$ and any arbitrary $\varepsilon \in \mathbf{R}_{+}$, belonging to a sampling sequence class $C_{J h}$. Also, there is an initial condition $y_{0}=$ $y_{0}(\varepsilon)$ which shadows each $\mathrm{O}\left(\widehat{x}_{J}, \Gamma, \varepsilon\right) \in C O\left(C_{J h}, \varepsilon\right)$ for each given $\rho, \varepsilon \in \mathbf{R}_{+}$with the perturbation fulfilling $\sum_{i=0}^{J-1} \bar{g}_{i}<$ $\varepsilon$.

Proof. One gets from (55) in Theorem 8 together with either (51) or (52) that

$$
\max _{t \in\left[t_{0}, t_{J}\right]}\|e(t)\| \leq \varepsilon=\left\|e\left(t_{0}\right)\right\|+\rho+\sum_{i=0}^{J-1} \bar{g}_{i}
$$

with any arbitrary real constant $0<\rho \leq \varepsilon-\sum_{i=0}^{J-1} \bar{g}_{i}$, provided that $\sum_{i=0}^{J-1} \bar{g}_{i}<\varepsilon$, and any given real constant $\varepsilon$ for an (shadowing) initial condition $y\left(t_{0}\right)$ of the true differential equation fulfilling

$$
\begin{aligned}
& \left|\left\|y\left(t_{0}\right)\right\|-\left\|x\left(t_{0}\right)\right\|\right| \\
& \quad \leq\left\|e\left(t_{0}\right)\right\| \\
& \quad \leq \min \left(\varepsilon-\rho-\sum_{i=0}^{J-1} \bar{g}_{i}, \frac{1-\sum_{i=0}^{J-1} h_{i} \lambda_{i}}{\sum_{i=0}^{J-1} h_{i} \lambda_{i}} \bar{g}\right) .
\end{aligned}
$$

Note that a sufficient condition guaranteeing (69) is

$$
\left\|e\left(t_{0}\right)\right\| \leq \min \left(\varepsilon-\varepsilon_{0}-J \bar{g}, \frac{1-\sum_{i=0}^{J-1} h_{i} \lambda_{i}}{\sum_{i=0}^{J-1} h_{i} \lambda_{i}} \bar{g}\right)
$$

since $\bar{g} \geq\left(\left(\sum_{i=0}^{J-1} h_{i} \lambda_{i}\right) /\left(1-\sum_{i=0}^{J-1} h_{i} \lambda_{i}\right)\right)\left\|e\left(t_{0}\right)\right\|$ from (56b) in Theorem 8. Thus, it suffices to take $0<\rho \leq \varepsilon$ to zero in either (39) or (40) in Theorem 6 to fix an admissible sampling sequence $\widehat{t}_{J}=\widehat{t}_{J}(\varepsilon)$ so as to get the result.

Remark 16. A particular case of Theorem 15 for the perturbations (42) which are defined only at sampling instants, which has been discussed in Theorem 7 , is obtained by the particular constraint below obtained from (72) and (73):

$$
\left\|e\left(t_{0}\right)\right\| \leq \varepsilon-\rho-J \bar{g} \leq \varepsilon-\rho-\sum_{i=0}^{J-1} \bar{g}_{i} .
$$

Remark 17. Note that the condition $\sum_{i=0}^{\infty} h_{i} \lambda_{i} \leq \chi<1$ of applicability in Theorems 8 and 15 when $J$ is infinity can be considered in certain cases when the perturbation vanishes asymptotically as, for instance, when it vanishes as an exponential rate.

\section{Simulation Examples}

This section contains two numerical examples regarding the theoretical results obtained in Sections 2 and 3.

Example 1. The first example is concerned with the nonlinear model describing the human heart rate during treadmill exercise [29], whose equations are given by

$$
\begin{gathered}
\dot{y}_{1}=-a_{1} y_{1}+a_{2} y_{2}, \\
\dot{y}_{2}=-a_{3} y_{2}+a_{4} \frac{y_{1}}{1+e^{-\left(\bar{c} y_{1}-a_{5}\right)}}
\end{gathered}
$$


with $\bar{c}=1, a_{1}=2.2, a_{2}=19.96, a_{3}=0.0831, a_{4}=0.002526$, and $a_{5}=8.32$. This model, with an external control input, has been used to design training protocols for patients with cardiovascular problems [29]. Figure 1 shows the evolution of this system with initial conditions $y_{1}(0)=y_{2}(0)=1$ on the time interval $[0,50]$ seconds. by

For the system (75), the nonlinear function $f(y)$ is given

$$
f(y)=f\left(y_{1}, y_{2}\right)=\left[\begin{array}{c}
-a_{1} y_{1}+a_{2} y_{2} \\
-a_{3} y_{2}+a_{4} \frac{y_{1}}{1+e^{-\left(\bar{c} y_{1}-a_{5}\right)}}
\end{array}\right] .
$$

The first step to apply the results stated in Section 2 and obtain a truncated approximate model for (75) is to verify that conditions (13a) and (13b) hold. One way to check this fact is to depict the norms of the state vector of the function $f(y)$ and of its derivative $f^{\prime}(y)$ and observe their behavior. Thus, the following Figures 2 and 3 show the time evolution of these norms. In particular, Figure 2 shows the values of the 2-norm of the state, $\|y\|_{2}$, and the function, $\|f(y)\|_{2}$. The supremum of these norms on this interval are $\sup _{t \in[0,50]}\|y\|_{2}=8.94$ and $\sup _{t \in[0,50]}\|f(y)\|_{2}=17.84$. On the other hand, Figure 3 shows the difference between the norm of the function, $\|f(y)\|_{2}$, and the norm of its derivative $\left\|f^{\prime}(y)\right\|_{2}$. As it can be appreciated, this difference is positive implying that the linear approximation of the function is always bounded by the function itself. The supremum of these two norms on this interval is $\sup _{t \in[0,50]}\|f(y)\|_{2}=17.84$ and $\sup _{t \in[0,50]}\left\|f^{\prime}(y)\right\|_{2}=17.76$. In this way, if we choose $K=$ 0.997 and $K_{1}=9$, we have

$$
\begin{gathered}
17.84=\sup _{t \in[0,50]}\|f(y)\|_{2}<0.997 \sup _{t \in[0,50]}\|y\|_{2}+9=17.91 \\
17.76=\sup _{t \in[0,50]}\left\|f^{\prime}(y)\right\|_{2}<0.997 \sup _{t \in[0,50]}\|f(y)\|_{2}=17.78 .
\end{gathered}
$$

Hence, it is corroborated that both (13a) and (13b) hold. Notice that from a practical point of view, the analytical determination of the constants $K$ and $K_{1}$ used in (13a) and (13b) is not necessary since a simple numerical experiment allows us to verify these upper-bounds. In consequence, the results stated in Section 2 can be applied in practical situations easily.

Once the basic conditions have been checked, a truncated approximate model (10) is generated for this problem by considering $\ell=1<2=n$. Thus, we have

$$
\dot{x}=f\left(x_{i}\right)+J\left(x_{i}\right)\left(x(t)-x_{i}\right),
$$

where

$$
\begin{gathered}
f^{\prime}\left(x_{i}\right)=J\left(x_{i}\right)=\left(\begin{array}{cc}
-a_{1} & a_{2} \\
J_{21} & -a_{3}
\end{array}\right), \\
J_{21}=\frac{a_{4}\left(1+e^{-\left(\bar{c} x_{i 1}-a_{5}\right)}\right)+a_{4} \bar{c} x_{i 1} e^{-\left(\bar{c} x_{i 1}-a_{5}\right)}}{\left(1+e^{-\left(\bar{c} x_{i 1}-a_{5}\right)}\right)^{2}}
\end{gathered}
$$

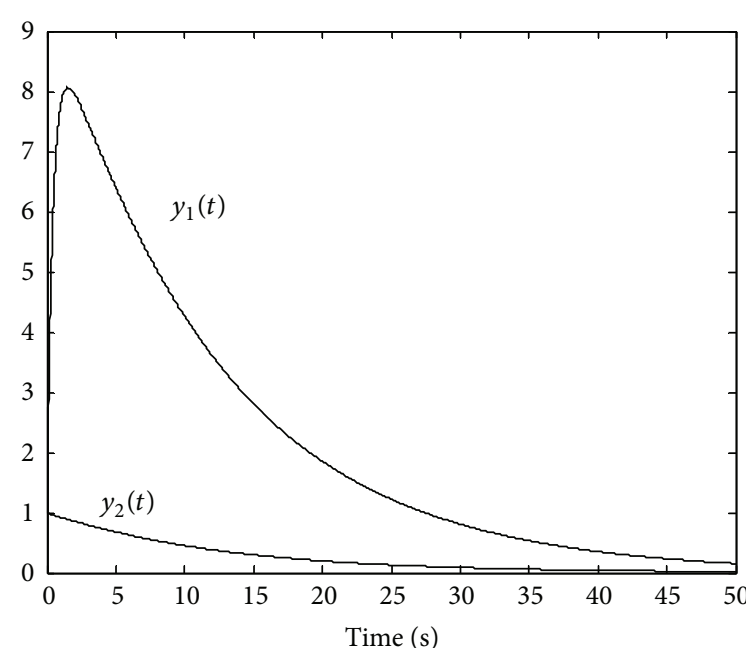

FIgURE 1: State evolution for the system (75).

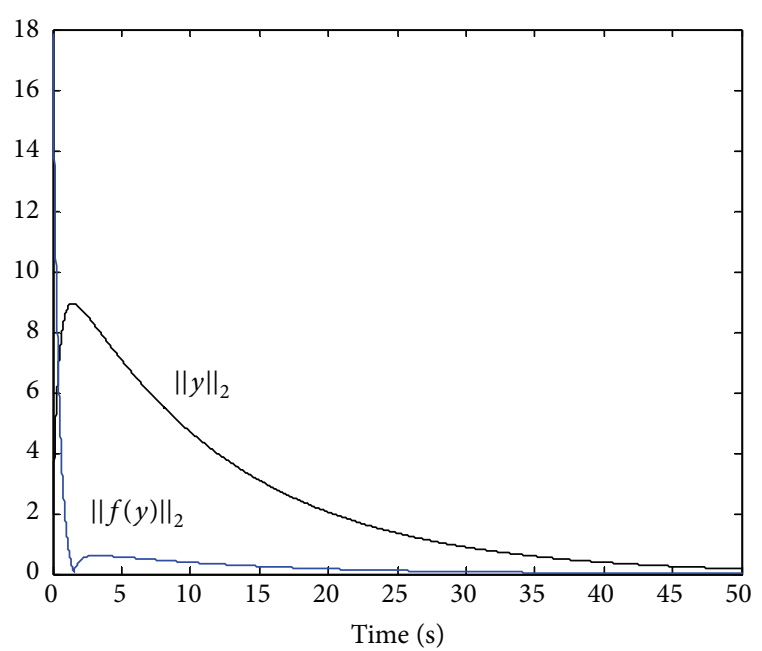

FIgURE 2: Relation between the 2-norms of the state, $y$, and the function, $f(y)$.

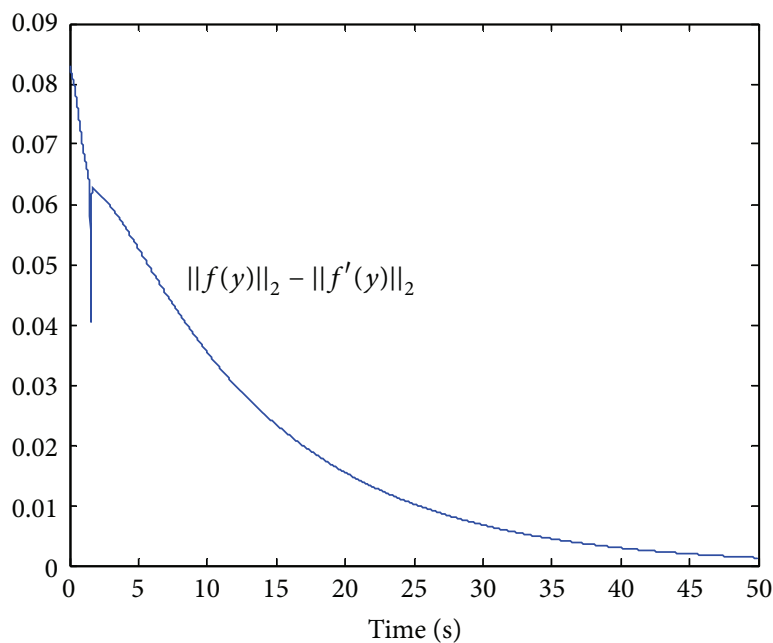

FIgURE 3: Relation between the 2-norms of the function, $f(y)$, and its derivative, $f^{\prime}(y)$. 


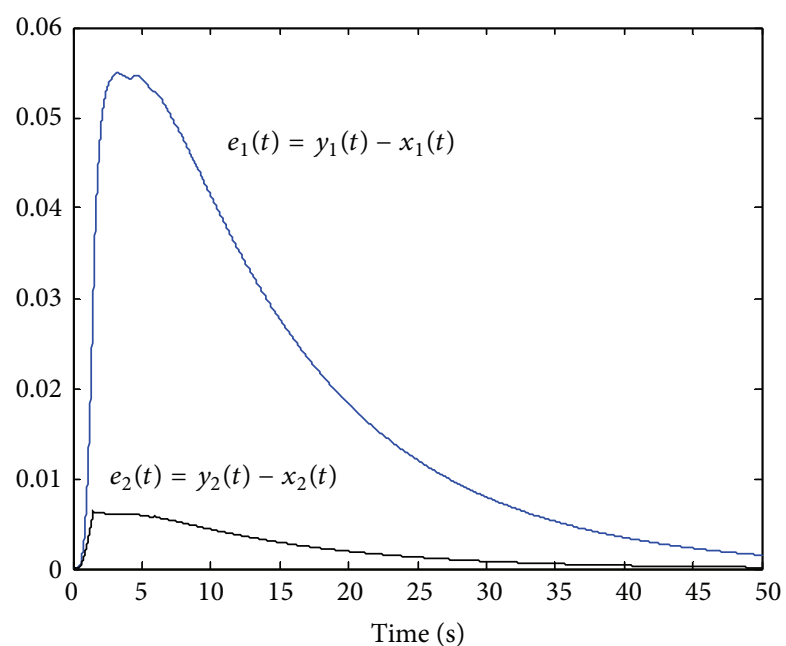

Figure 4: Error between the actual system and the approximate model.

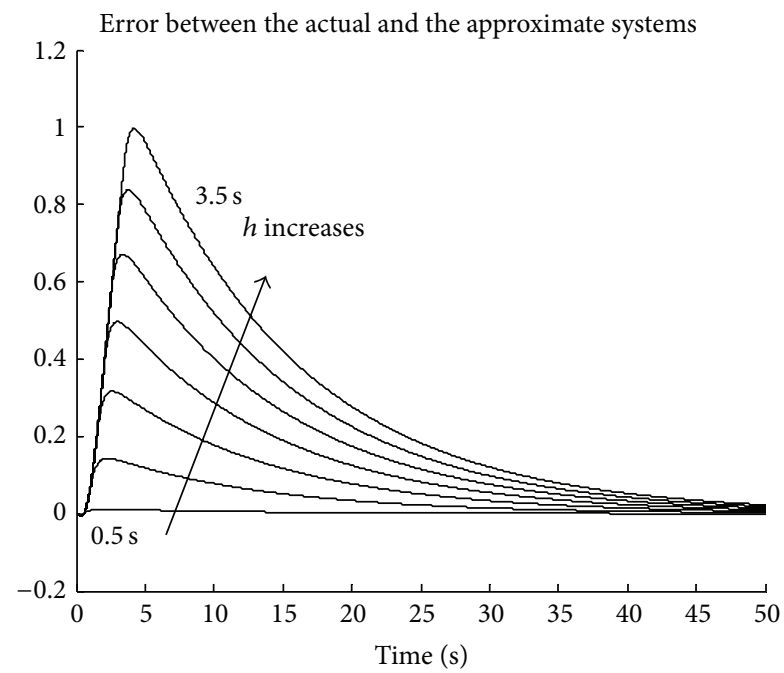

FIGURE 5: Effect of the variation of the sampling time, $h$, in the error of the first state variable, $x_{1}(t)$.

with initial conditions $y_{1}(0)=y_{2}(0)=x_{1}(0)=x_{2}(0)=1$. The sampling instants $\left\{t_{i}\right\}$ have been chosen uniformly in time as $t_{i}-t_{i-1}=h=1.5 \mathrm{~s}$. The error between the actual and the approximate model with this sampling time is depicted in Figure 4.

As it can be deduced from Figure 4, the error is very low and, therefore, the exact solution is shadowed by the solution of the approximate model. An important feature appears at this point which is how we should select the sampling time. Lemma 4 and Theorem 6 contain the analytical results providing the formal background on how to select it. However, from a practical point of view a trial-error procedure can be employed to obtain an appropriate sampling time. Thus, Figures 5 and 6 show how a variation in the sampling time impacts the error between the complete system and the approximate model. Figure 5 displays the impact on the first

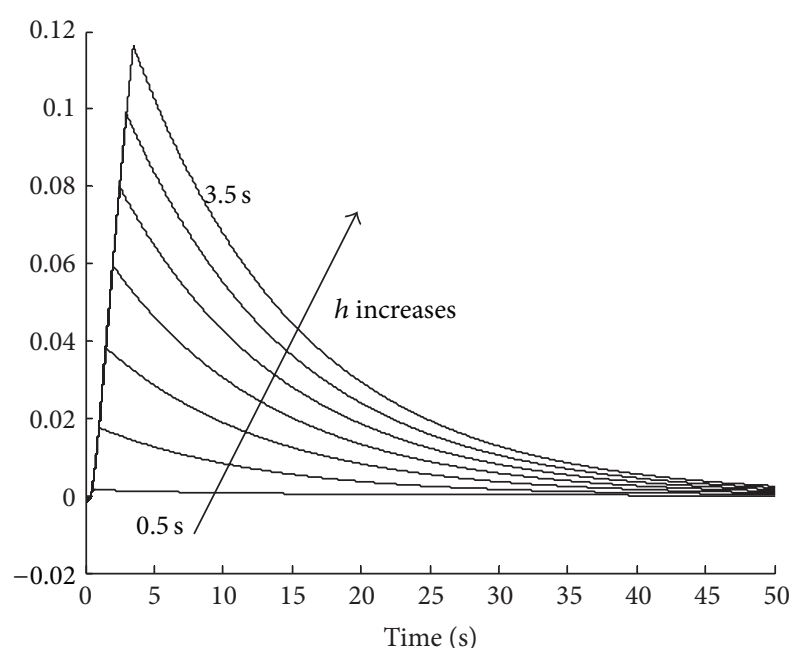

FIGURE 6: Effect of the variation of the sampling time, $h$, in the error of the second state variable, $x_{2}(t)$.

state variable while Figure 6 depicts the influence in the second one. As it is displayed in Figures 5 and 6, the larger the sampling time is, the larger the error between both systems is, as well. Hence, if we fix an upper-bound for the desired error, we may start with a tentative value for the sampling time and increase it if the maximum of the error is below that threshold or decrease it if the error exceeds the desired bound. This procedure allows us to tune an appropriate sampling time by just conducting a series of numerical experiments. Therefore, the mathematical results presented in Section 2 can be applied in a practical way with little effort since the computation of the bounds is not explicitly necessary to construct the approximate truncated model. Afterwards, this approximate model could be used for simulation or control design purposes. For instance, the obtained affine model could simplify the design of the controller with respect to the case when the original nonlinear model is used.

Example 2. The second example is related to the Van der Pol equation which exhibits a limit cycle as it is widely known. The equations are given by

$$
\begin{gathered}
\dot{y}_{1}=y_{2}, \\
\dot{y}_{2}=\mu\left(1-y_{1}^{2}\right) y_{2}-y_{1}
\end{gathered}
$$

with $\mu=1$, output $z(t)=y_{1}(t)$, initial conditions $y_{1}(0)=4$ and $y_{2}(0)=-0.5$, and

$$
f(y)=f\left(y_{1}, y_{2}\right)=\left[\begin{array}{c}
y_{2} \\
\mu\left(1-y_{1}^{2}\right) y_{2}-y_{1}
\end{array}\right] .
$$

The phase portrait of the Van der Pol equation is depicted in Figure 7.

In this case, the results introduced in Section 3 regarding the error between the actual and the approximate model in the presence of bounded perturbations will be used as a tool to analyze the stability of the limit cycle. Thus, 


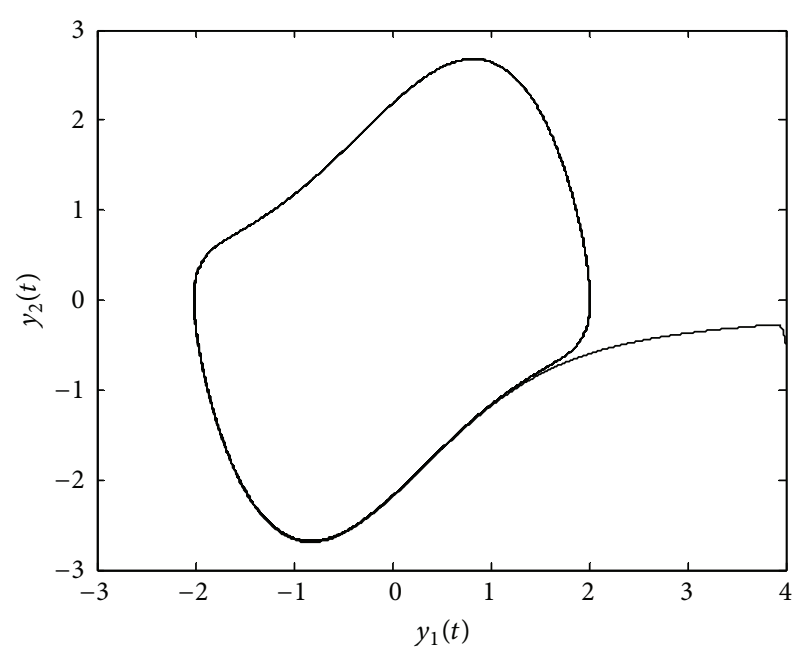

FIgure 7: Phase portrait of the Van der Pol equation.

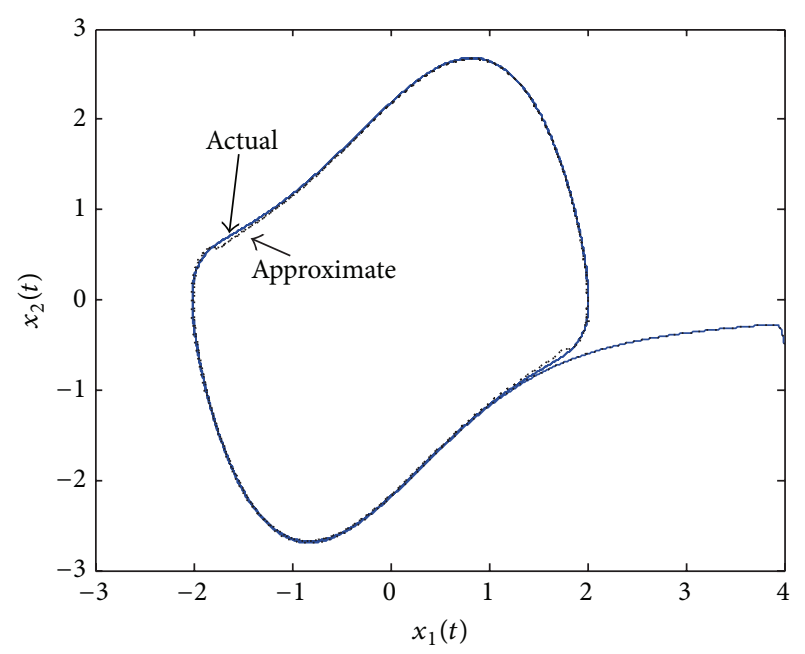

FIGURE 8: Phase portrait of the actual and approximate systems.

the approximate truncated model is given with $\ell=1<2=n$ by

$$
\dot{x}=f\left(x_{i}\right)+J\left(x_{i}\right)\left(x(t)-x_{i}\right),
$$

where

$$
\begin{gathered}
f^{\prime}\left(x_{i}\right)=J\left(x_{i}\right)=\left(\begin{array}{cc}
0 & 1 \\
J_{21} & J_{22}
\end{array}\right), \\
J_{21}=-2 \mu x_{i 1} x_{i 2}-1, \\
J_{21}=\mu-\mu x_{i 1}^{2} .
\end{gathered}
$$

In this example, the sampling points $x_{i}$ will be generated by using the constant amplitude difference sampling criterion (CADSC) introduced in [4] as a method to generate sampling points in discretization procedures. This method is proposed as a way to generate the sequence of sampling points in a practical way, which shows that the application of the presented theories to real problems is feasible. Thus, the CADSC

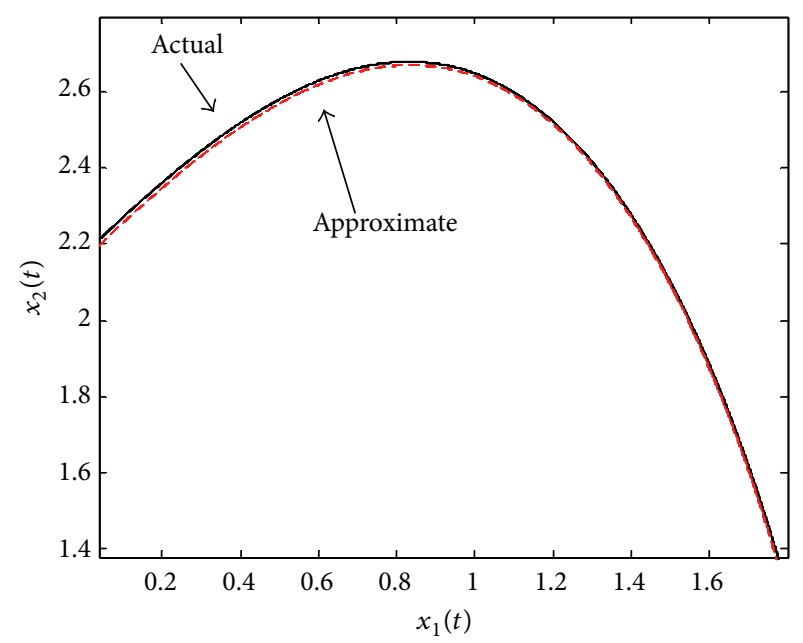

FIGURE 9: Zoom on the phase portrait of the actual and approximate systems.

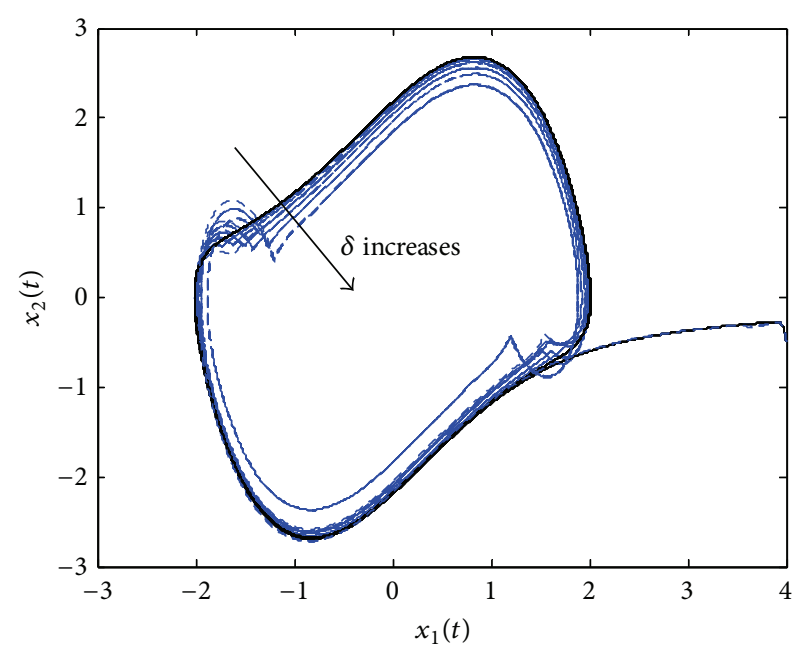

FIGURE 10: Effect of the variation of the sampling threshold $\delta$ in the approximate model.

method proposes to generate a new sampling point when the continuous-time output differs from the previous sampled one a certain threshold. Mathematically,

$$
t_{i+1}=\arg \min \left(R_{0^{+}} \ni t>t_{i}:\left|x_{1}(t)-x_{1}\left(t_{i}\right)\right|=\delta_{i} \in R_{+}\right),
$$

where $\delta_{i}$ denotes the variation threshold. For this example, consider a constant threshold with a value of $\delta_{i}=\delta=0.15$. Figures 8 and 9 display the solution of the actual and the approximate systems.

It can be appreciated in Figures 8 and 9 that the solution of the actual system is shadowed by one of the approximate models, confirming the results stated in Proposition 14. As the threshold $\delta$ on the sampling criterion enlarges, the sampling takes place in a more separate way, a fact that degrades the quality of the approximate solution as Figure 10 reveals. 


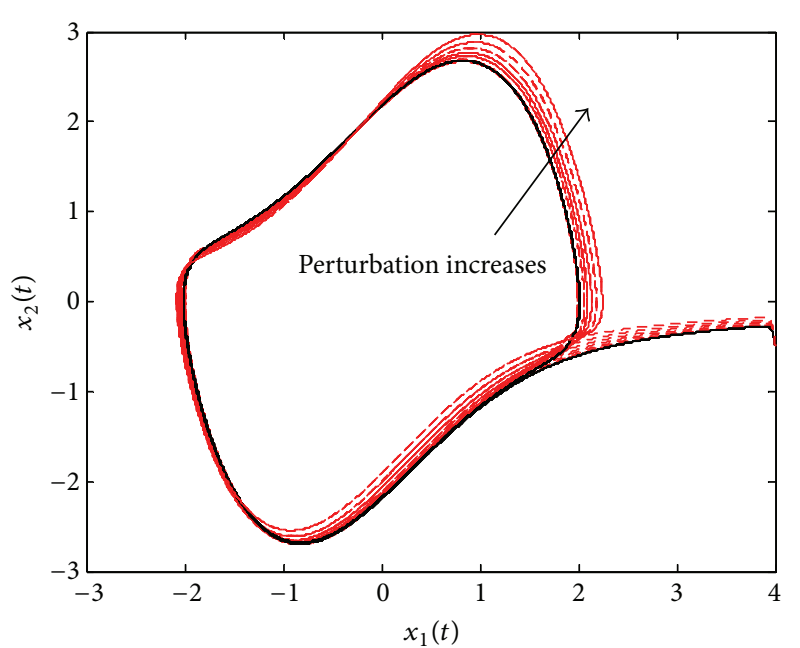

FIGURE 11: Stability of the limit cycle under increasing perturbations.

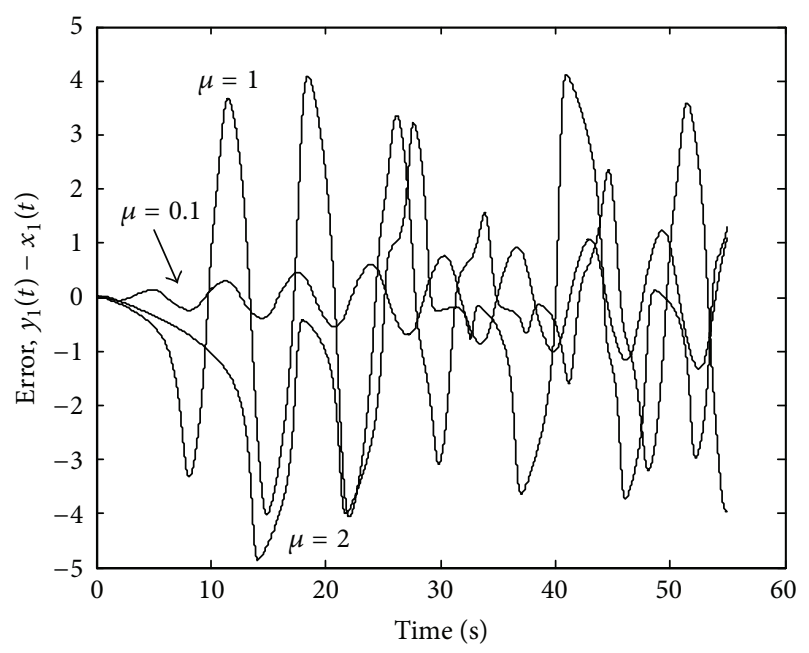

Figure 12: Error between $y_{1}(t)$ and $x_{1}(t)$ for different values for $\mu$.

Figure 10 shows that the larger the threshold is, the smaller the approximation capabilities of the truncated model is. This feature is due to the fact that a larger threshold implies a greater separation between the sampling points. In Figure $10, \delta$ is modified from 0.15 to 0.4 . Thus, as Lemma 4 states, a large intersampling period might lead to higher errors in the approximated model. At this point we can introduce a bounded perturbation $g\left(t_{i}\right)$ at sampling points to analyze the stability of the limit cycle. For this, we can firstly select a value for the threshold $\delta$ in such a way that the solution of the approximate model shadows the one of the actual system. Afterwards, we can apply different perturbations to the system in an increasing way. If the limit cycle preserves its shape under this scheme, this would indicate that it is stable. This procedure has been applied in Figure 11.

Since the shape of the limit cycle is maintained, the stability of the original system is deduced from one of the approximate truncated models. Moreover, the shadowing

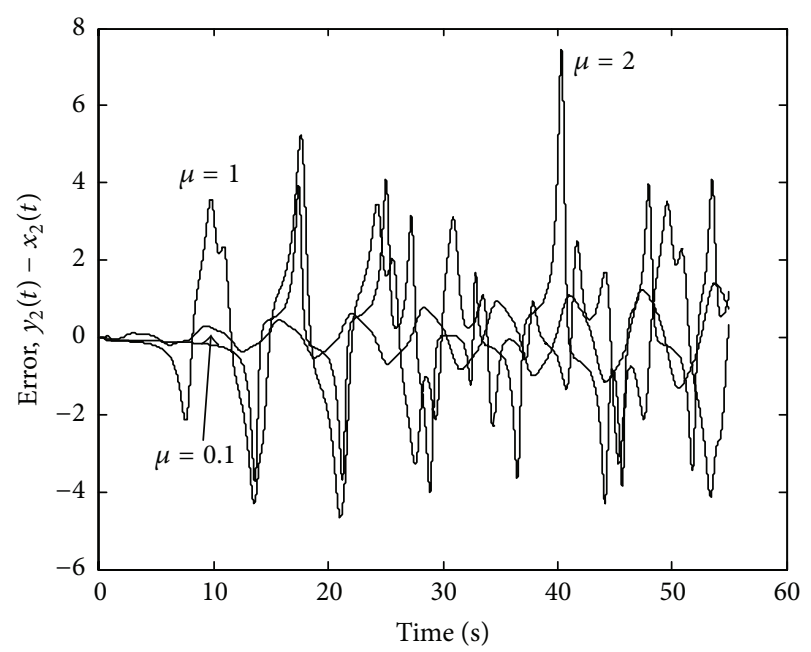

Figure 13: Error between $y_{2}(t)$ and $x_{2}(t)$ for different values for $\mu$.

property can be interpreted in terms of stability of the limit cycle in the following way. If the error between the solutions of the actual system and the approximate one under the same perturbation is less in a system $A$ than in another system $B$, this means that $A$ is more stable than $B$. Thus, the shadowing property can be viewed as a concept to measure the relative stability of systems by using its Taylor series expansion and construction of approximate models. For instance, consider the van del Pol equation with three different values of $\mu \epsilon$ $\{0.1,1,2\}$. The behavior of the van der Pol equation depends on the value of $\mu$ as it is widely recognized. Thus, the approximate perturbed model can be used to compute the error between the actual and reduced models in each case and determine for which value of $\mu$ the Van der Pol equation is "more stable," that is, has a greater relative stability. Thus, we fix the perturbation amplitude to $g\left(t_{i}\right)=0.35$ and carry out some numerical experiments with the different values for $\mu$. In this way, Figure 12 displays the error in the first state variable between the actual and approximate models while Figure 13 shows the error in the second state variable for each value of $\mu$.

Figures 12 and 13 show that the larger $\mu$ is, the larger the peak error is. Therefore, in this case, systems with smaller $\mu$ have a greater relative stability. Finally, this approximate affine model could be used, as in the previous example, to design a control system based on a reduced model, rather than using the complete nonlinear one. In conclusion, the results presented in the previous sections have been applied in some case studies with little effort, a fact that backs up its potential practical applications.

\section{Conflict of Interests}

The authors declare that there is no conflict of interests regarding the publication of this paper. 


\section{Acknowledgments}

The authors are very grateful to the Spanish Government for its support of this research through Grant DPI2012-30651 and to the Basque Government for its support of this research through Grants IT378-10 and SAIOTEK S-PE12UN015. They are also grateful to the University of Basque Country for its financial support through Grant UFI 2011/07.

\section{References}

[1] M. de la Sen, "Application of the nonperiodic sampling to the identifiability and model matching problems in dynamic systems," International Journal of Systems Science, vol. 14, no. 4, pp. 367-383, 1983.

[2] M. de la Sen, "Stability of switched feedback time-varying dynamic systems based on the properties of the gap metric for operators," Abstract and Applied Analysis, vol. 2012, Article ID 612198, 17 pages, 2012.

[3] M. de la Sen, "About the stabilization of a nonlinear perturbed difference equation," Discrete Dynamics in Nature and Society, vol. 2012, Article ID 320302, 19 pages, 2012.

[4] M. De la Sen, J. C. Soto, and A. Ibeas, "Stability and limit oscillations of a control event-based sampling criterion," Journal of Applied Mathematics, vol. 2012, Article ID 684292, 25 pages, 2012.

[5] R. Gu, "The average-shadowing property and topological ergodicity," Journal of Computational and Applied Mathematics, vol. 206, no. 2, pp. 796-800, 2007.

[6] R. Gu, "On ergodicity of systems with the asymptotic average shadowing property," Computers \& Mathematics with Applications, vol. 55, no. 6, pp. 1137-1141, 2008.

[7] R. Gu, "Recurrence and the asymptotic pseudo-orbit tracing property," Nonlinear Analysis: Theory, Methods \& Applications, vol. 66, no. 8, pp. 1698-1706, 2007.

[8] K. Lee and K. Sakai, "Various shadowing properties and their equivalence," Discrete and Continuous Dynamical Systems A, vol. 13, no. 2, pp. 533-540, 2005.

[9] E. S. van Vleck, "Numerical shadowing using componentwise bounds and a sharper fixed point result," SIAM Journal on Scientific Computing, vol. 22, no. 3, pp. 787-801, 2000.

[10] S. Reich, "Backward error analysis for numerical integrators," SIAM Journal on Numerical Analysis, vol. 36, no. 5, pp. 15491570, 1999.

[11] T. M. Apostol, Mathematical Analysis, Addison-Wesley, Reading, Mass, USA, 1958.

[12] Q. L. Zhang, G. Z. Dai, J. Lam, L. Q. Zhang, and M. De La Sen, "Asymptotic stability and stabilization of descriptor systems," Acta Automatica Sinica, vol. 24, no. 2, pp. 208-211, 1998.

[13] M. de la Sen, "Fundamental properties of linear control systems with after-effect. I. The continuous case," Mathematical and Computer Modelling, vol. 10, no. 7, pp. 473-489, 1988.

[14] R. A. Maher and R. Samir, "Robust stability of a class of unstable systems under mixed uncertainty," Journal of Control Science and Engineering, vol. 2011, Article ID 970962, 8 pages, 2011.

[15] D. Boutat, "Extended nonlinear observer normal forms for a class of nonlinear dynamical systems," International Journal of Robust and Nonlinear Control, 2013.

[16] M. Benchohra and M. Ziane, "Impulsive evolution inclusions with state-dependent delay and multivalued jumps," Electronic
Journal of Qualitative Theory of Differential Equations, no. 42, pp. 1-21, 2013.

[17] C. Tunç and M. Ateş, "Boundedness of solutions to differential equations of fourth order with oscillatory restoring and forcing terms," Discrete Dynamics in Nature and Society, vol. 2013, Article ID 758796, 5 pages, 2013.

[18] C. Tunc and M. Gozen, "Stability and uniform boundedness in multidelay functional differential equations of third order," Abstract and Applied Analysis, vol. 2013, Article ID 248717, 7 pages, 2013.

[19] J. Diblík, M. Fečkan, and M. Pospíšil, "Representation of a solution of the Cauchy problem for an oscillating system with multiple delays and pairwise permutable matrices," Abstract and Applied Analysis, vol. 2013, Article ID 931493, 10 pages, 2013.

[20] B. Baculikova, J. Dzurina, and Y. V. Rogovchenko, "Oscillation of third order trinomial delay differential equations," Applied Mathematics and Computation, vol. 218, no. 13, pp. 7023-7033, 2012.

[21] J. Baštinec, L. Berezansky, J. Diblík, and Z. Šmarda, "On the critical case in oscillation for differential equations with a single delay and with several delays," Abstract and Applied Analysis, vol. 2010, Article ID 417869, 20 pages, 2010.

[22] M. Hasanbulli and Y. V. Rogovchenko, "Oscillation criteria for second order nonlinear neutral differential equations," Applied Mathematics and Computation, vol. 215, no. 12, pp. 4392-4399, 2010.

[23] Z. Han, Y. Zhao, Y. Sun, and C. Zhang, "Oscillation for a class of fractional differential equation," Discrete Dynamics in Nature and Society, vol. 2013, Article ID 390282, 6 pages, 2013.

[24] J. C. Soto and M. Delasen, "Nonlinear oscillations in nonperiodic sampling systems," Electronics Letters, vol. 20, no. 20, pp. 816-818, 1984.

[25] M. de la Sen, "Oscillatory behavior in linear difference equations under unmodeled dynamics and parametrical errors," Mathematical Problems in Engineering, vol. 2007, Article ID 25692, 18 pages, 2007.

[26] J. C. Soto and M. Delasen, "On the derivation and analysis of a non-linear model for describing a class of adaptive sampling laws," International Journal of Control, vol. 42, no. 6, pp. 13471368, 1985.

[27] J. Chacón, J. Sánchez, A. Visioli, L. Yebra, and S. Dormido, "Characterization of limit cycles for self-regulating and integral processes with PI control and send-on-delta sampling," Journal of Process Control, vol. 23, no. 6, pp. 826-838, 2013.

[28] R. Memarbashi and H. Rasuli, "Notes on the dynamics of nonautonomous discrete dynamical systems," Journal of Advanced Research in Dynamical and Control Systems, vol. 6, no. 2, pp. 8-17, 2014.

[29] F. Mazenc, M. Malisoff, and M. de Querioz, "Tracking control and robustness analysis for a nonlinear model of human heart rate during exercise," Automatica, vol. 47, no. 5, pp. 968-974, 2011. 


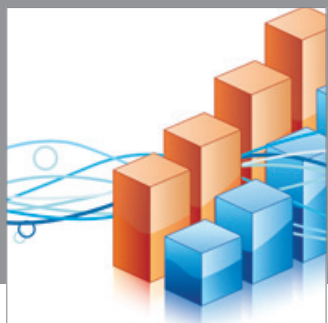

Advances in

Operations Research

mansans

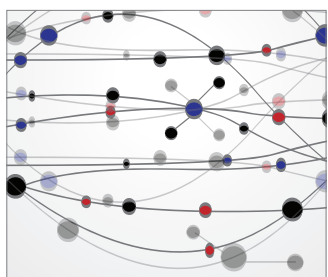

The Scientific World Journal
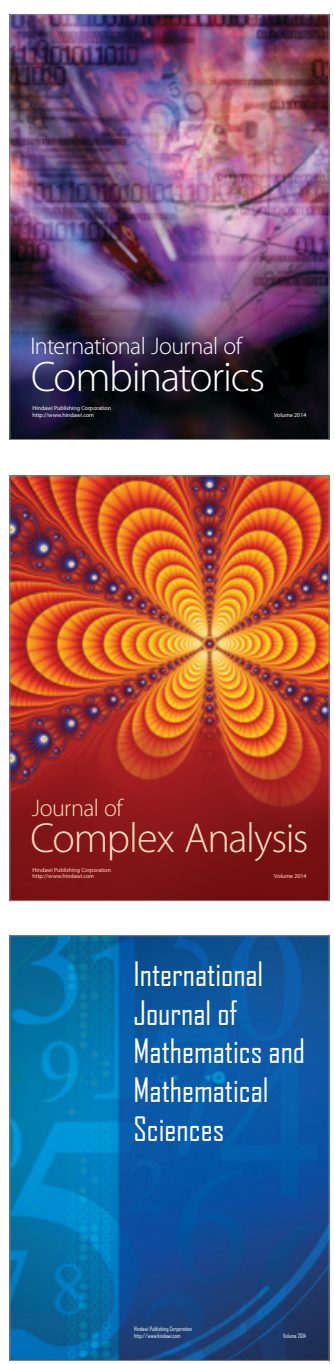
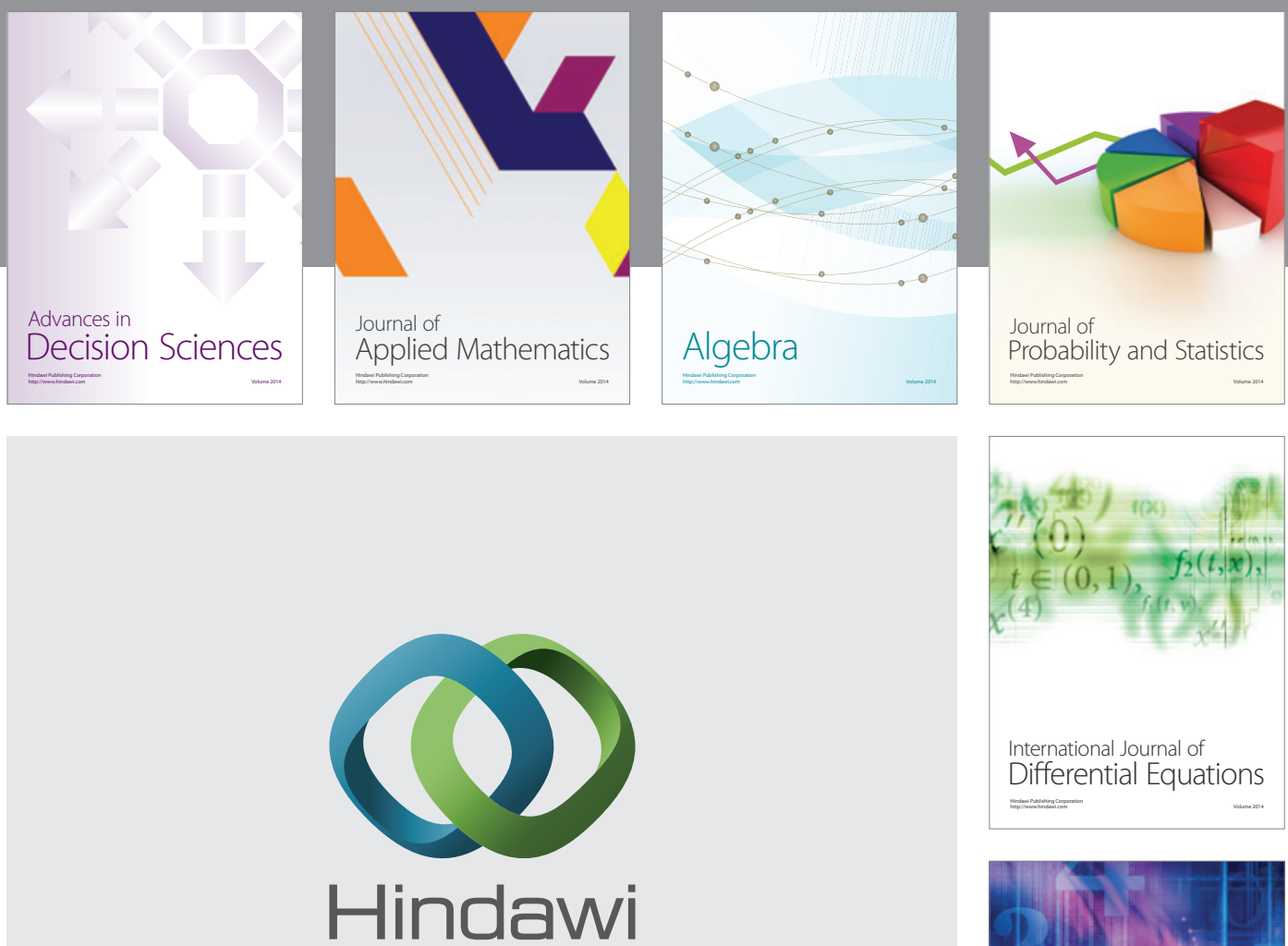

Submit your manuscripts at http://www.hindawi.com
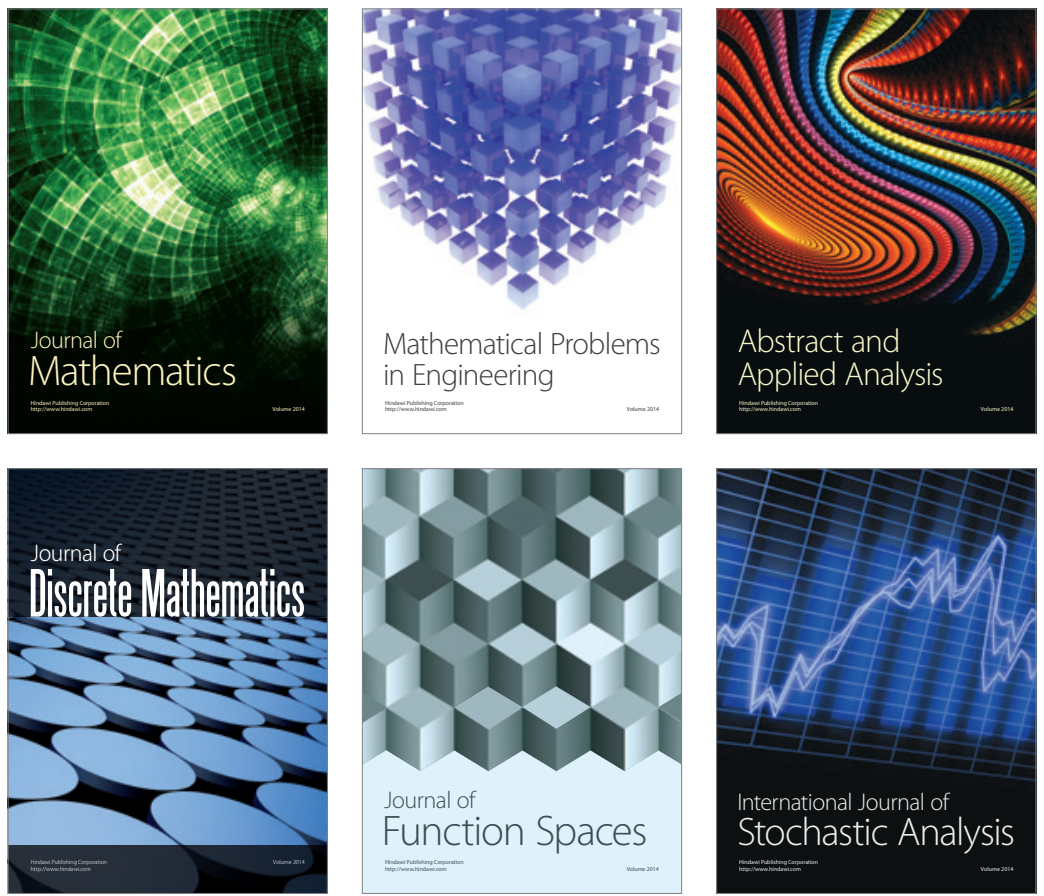

Journal of

Function Spaces

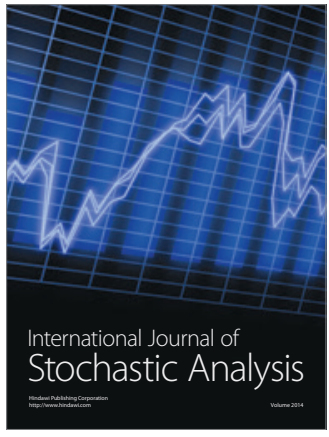

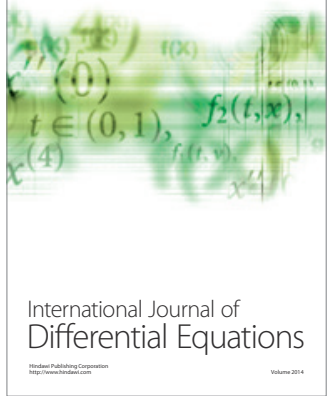
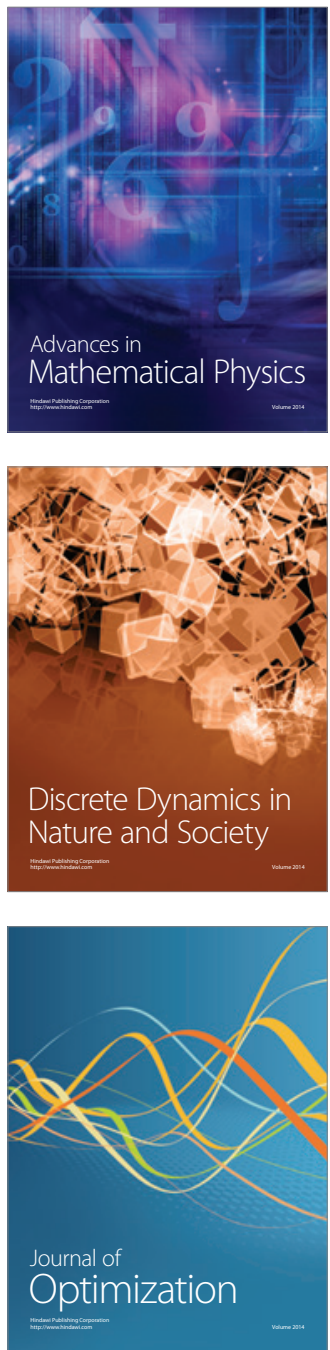